\title{
The rigidity of crystalline color superconducting quark matter
}

\author{
Massimo Mannarelli, ${ }^{1, *}$ Krishna Rajagopal, ${ }^{1, \dagger}$ and Rishi Sharma ${ }^{1, \text { t }}$ \\ ${ }^{1}$ Center for Theoretical Physics, Massachusetts Institute of Technology, Cambridge, MA 02139
}

(Dated: August 7, 2007)

\begin{abstract}
We calculate the shear modulus of crystalline color superconducting quark matter, showing that this phase of dense, but not asymptotically dense, three-flavor quark matter responds to shear stress like a very rigid solid. To evaluate the shear modulus, we derive the low energy effective Lagrangian that describes the phonons that originate from the spontaneous breaking of translation invariance by the spatial modulation of the gap parameter $\Delta$. These massless bosons describe space- and time-dependent fluctuations of the crystal structure and are analogous to the phonons in ordinary crystals. The coefficients of the spatial derivative terms of the phonon effective Lagrangian are related to the elastic moduli of the crystal; the coefficients that encode the linear response of the crystal to a shearing stress define the shear modulus. We analyze the two particular crystal structures which are energetically favored over a wide range of densities, in each case evaluating the phonon effective action and the shear modulus up to order $\Delta^{2}$ in a Ginzburg-Landau expansion, finding shear moduli which are 20 to 1000 times larger than those of neutron star crusts. The crystalline color superconducting phase has long been known to be a superfluid — by picking a phase its order parameter breaks the quark-number $U(1)_{B}$ symmetry spontaneously. Our results demonstrate that this superfluid phase of matter is at the same time a rigid solid. We close with a rough estimate of the pinning force on the rotational vortices which would be formed embedded within this rigid superfluid upon rotation. Our results raise the possibility that (some) pulsar glitches could originate within a quark matter core deep within a neutron star.
\end{abstract}

PACS numbers: $12.38 . \mathrm{Mh}, 24.85 .+\mathrm{p}$

\section{INTRODUCTION}

Quantum chromodynamics (QCD) predicts that if one can squeeze nuclear matter to the point that the number of quarks per unit volume is greater than that within a nucleon, the matter that results cannot be described as made up of nucleons: the individual nucleons are crushed into quark matter [1]. It remains an open question whether "liberated" quark matter exists at the core of neutron stars, which have central densities at most $\sim 10$ times that of ordinary nuclear matter. One route to answering this question would be an ab initio calculation of the range of densities at which the expected first order transition from nuclear to quark matter occurs in QCD. No currently available calculational method promises such a result any time soon, which is not surprising since locating such a first order transition requires quantitative control of the pressure of both phases, making this a much more difficult question

\footnotetext{
*Electronic address: massimo@lns.mit.edu

${ }^{\dagger}$ Electronic address: krishna@lns.mit.edu

${ }^{\ddagger}$ Electronic address: sharma@mit.edu
}

than understanding the properties of either phase on its own. The more promising route to answering whether neutron stars feature quark matter cores requires first understanding the qualitative and semiquantitative properties of quark matter and then discerning how its presence deep within a neutron star would affect astronomically observed or observable phenomena.

Except during the first few seconds after their birth, the temperature of neutron stars is expected to be of the order of tens of $\mathrm{keV}$ at most. At such low temperatures, if deconfined quark matter exists in the core of neutron stars it is likely to be in one of the possible color superconducting phases, whose critical temperatures are generically of order tens of $\mathrm{MeV}$ [2]. Furthermore, since compact star temperatures are so much smaller than these critical temperatures, for many purposes (including in particular for a calculation of its rigidity) the quark matter that may be found within neutron stars is well-approximated as having zero temperature, as we shall assume throughout.

The essence of color superconductivity is quark pairing, driven by the BCS mechanism which operates whenever there is an attractive interaction between fermions at a Fermi surface [3]. The QCD 
quark-quark interaction is strong and attractive between quarks that are antisymmetric in color, so we expect cold dense quark matter to generically exhibit color superconductivity. It is by now well established that at asymptotic densities, where the up, down and strange quarks can be treated on an equal footing and the disruptive effects of the strange quark mass can be neglected, quark matter is in the color flavor locked (CFL) phase [2, 4]. The CFL condensate is antisymmetric in color and flavor indices and therefore involves pairing between quarks of different colors and flavors.

To describe quark matter as may exist in the cores of compact stars, we need to consider quark number chemical potentials $\mu$ of order $500 \mathrm{MeV}$ at most (corresponding to $\mu_{B}=3 \mu$ of order $1500 \mathrm{MeV}$ ). The relevant range of $\mu$ is low enough that the strange quark mass, $M_{s}$, lying somewhere between its current mass of order $100 \mathrm{MeV}$ and its vacuum constituent mass of order $500 \mathrm{MeV}$, cannot be neglected. Furthermore, bulk matter, if present in neutron stars, must be in weak equilibrium and must be electrically and color neutral. All these factors work to separate the Fermi momenta of the quarks by $\sim M_{s}^{2} / \mu$, and thus disfavor the cross-species BCS pairing which characterizes the CFL phase. If we imagine beginning at asymptotically high densities and reducing the density, and suppose that CFL pairing is disrupted by the heaviness of the strange quark before color superconducting quark matter is superseded by baryonic matter, the CFL phase must be replaced by some other superconducting phase of quark matter which has less, and less symmetric, pairing.

Considering only homogeneous condensates, the next phase down in density is the gapless CFL (gCFL) phase [5, 6, 7, 8, 9, 10, 11, 12, 13]. In this phase, quarks of all three colors and all three flavors still form ordinary Cooper pairs, but there are regions of momentum space in which certain quarks do not succeed in pairing, and these regions are bounded by momenta at which certain fermionic quasiparticles are gapless. This variation on BCS pairing - in which the same species of fermions that pair feature gapless quasiparticles - was first proposed for two flavor quark matter [14] and in an atomic physics context [15]. In all these contexts, however, the gapless paired state turns out in general to suffer from a "magnetic instability": it can lower its energy by the formation of counter-propagating currents [16, 17].

In Refs. [18, 19, 20] the possibility that the next superconducting phase down in density has a crystalline structure was explored. (For other possible patterns of three-flavor and three-color pairing, see Refs. 21, 22]. For possibilities in which only two colors of quarks pair, see Refs. 23]. For possibilities in which only quarks of the same flavor pair, see Refs. [24]. See Ref. 25] for an exhaustive study of less symmetric phases with BCS pairing only, the simplest example being the 2SC phase [26, 27], indicating that none are favorable.) Crystalline color superconductivity - the QCD analogue of a form of non-BCS pairing first considered by Larkin, Ovchinnikov, Fulde and Ferrell (LOFF) [28] - is an attractive candidate phase in the intermediate density regime 229, 30, 31, 32, 33, 34], because it allows quarks living on split Fermi surfaces to pair with each other. It does so by allowing Cooper pairs with nonzero total momentum $2 \mathbf{q}^{a}$, for some set of $\mathbf{q}^{a}$ 's whose magnitude is fixed and is of order the splitting between Fermi surfaces, and whose directions must be determined. In position space, this corresponds to condensates that vary in space like $\sum_{a} \exp \left(2 i \mathbf{q}^{\mathbf{a}} \cdot \mathbf{x}\right)$, meaning that the $\mathbf{q}^{a}$ 's are the reciprocal vectors which define the crystal structure of the condensate which is modulated periodically in space and therefore spontaneously breaks space translation invariance. These phases seem to be free from magnetic instability [35], which is consistent with the result that many of them have free energies that are lower than that of the (unstable) gCFL phase for wide ranges of parameter values [20].

Two crystal structures for three-flavor crystalline color superconducting quark matter have been identified as having particularly low free energy [20]. In the first, called the $2 \mathrm{Cube} 45 \mathrm{z}$ phase, the $\langle u d\rangle$ and $\langle u s\rangle$ condensates each form face-centered cubes in position space. (In momentum space, each has eight $\mathbf{q}^{a}$ vectors pointing toward the corners of a cube, which is the Bravais lattice of a face-centered cube.) The $\langle u s\rangle$ cube is rotated relative to the $\langle u d\rangle$ cube by 45 degrees. In the second favorable crystal structure, called the CubeX phase, the $\langle u d\rangle$ and $\langle u s\rangle$ condensates each describe pairing with four different wave vectors $\mathbf{q}^{a}$, with the eight $\mathbf{q}^{a}$ 's together forming the Bravais lattice of a single face-centered cube. The lattice spacing for these crystal structures is many tens of fm, as we shall discuss further in Section II, where we specify these crystal structures precisely.

These phases were identified upon making two crucial approximations [20], which we shall also employ. First, the QCD interaction between quarks 
was replaced by a point-like Nambu Jona-Lasinio (NJL) interaction with the quantum numbers of single gluon exchange. This is a conservative approach to the study of crystalline color superconductivity, since a point-like interaction tends to disfavor crystalline color superconductivity relative to a forwardscattering dominated interaction like single gluon exchange [33]. Second, the calculation employed a Ginzburg-Landau expansion in powers of the gap parameter $\Delta$ even though the values of $\Delta$ found in the calculation are large enough that the control parameter for this expansion, $\Delta /\left(M_{s}^{2} / 8 \mu\right)$, is about $1 / 2$, meaning that the expansion is pushed to or beyond the boundary of its regime of quantitative validity. Upon making these approximations, one or other of the two most favorable crystalline color superconducting phases is favored (i.e. has lower free energy than unpaired quark matter or any spatially uniform paired phase including the CFL and gCFL phases) for [20]

$$
2.9 \Delta_{0}<\frac{M_{s}^{2}}{\mu}<10.4 \Delta_{0} .
$$

Here, $\Delta_{0}$ is the gap parameter in the CFL phase with $M_{s}=0$, which is expected to lie somewhere between 10 and $100 \mathrm{MeV}$ [2]. The estimate (11) is valid upon assuming $\Delta \ll M_{s}^{2} / 8 \mu, \Delta_{0} \ll \mu$ and $M_{s}^{2} \ll \mu^{2}$, which here means $10.4 \Delta_{0}<\mu$. In reality, both $M_{s}$ and $\Delta_{0}$ are functions of $\mu$, and the regime (1) translates into a window in $\mu$. See Ref. [36] for an analysis in which both $M_{s}(\mu)$ and $\Delta_{0}(\mu)$ are calculated self-consistently in an NJL model, confirming that the window in $\mu$ corresponding to (1) is broad indeed. Even though it may not be quantitatively reliable, the breadth of the regime (1), corresponding to the remarkable robustness of the two most favorable crystalline phases which can be understood on qualitative grounds [20], makes it plausible that quark matter at densities accessible in neutron stars, say with $\mu \sim 350-500 \mathrm{MeV}$, will be in a crystalline phase. (Unless $\Delta_{0}$ lies at the upper end of its allowed range, in which case quark matter at accessible densities will be in the CFL phase.)

In this paper, we study the elastic properties of the CubeX and the 2Cube45z crystalline phases of three-flavor quark matter. We evaluate the shear moduli of these two structures by computing, in the Ginzburg-Landau approximation, the effective Lagrangian for the phonon modes which emerge due to the spontaneous breaking of translation invariance by the crystalline condensates. (See Ref. 31] for an analysis of phonons in 2-flavor crystalline color superconducting phases.) The shear modulus is related to the coefficients of the spatial derivative terms that appear in the phonon effective Lagrangian. We find that when these two crystalline phases are subject to shear stresses, they behave like rigid solids with shear moduli between 20 and 1000 times larger than those characteristic of conventional neutron star crusts.

We evaluate the shear modulus to order $\Delta^{2}$ in the Ginzburg-Landau expansion; higher order terms are suppressed only by powers of $\left[\Delta /\left(M_{s}^{2} / 8 \mu\right)\right]^{2}$, which is of order $1 / 4$ in the most favorable crystalline phases, large enough that significant higher order corrections to the shear modulus can be expected. However, as we shall discuss below all that is required from the calculation of the shear modulus in making the case that pulsar glitches could originate in a crystalline quark matter neutron star core is confidence that the shear modulus is comparable to or greater than that of the conventional neutron star crust. Since we find shear moduli that meet this criterion by a factor of 20 to 1000 , there is currently little motivation for evaluating higher order corrections.

The possibility that quark matter could occur in a solid phase has been raised previously by $\mathrm{Xu}$ [40]. He and his collaborators have explored some astrophysical consequences of a speculation that the quarks themselves could somehow be arranged in a crystalline lattice. The crystalline color superconducting phase is very different in character: being a superfluid, the quarks are certainly not frozen in place. Instead, what forms a crystalline pattern is the magnitude of the pairing condensate. Although it was clear prior to the present work that in this phase translational invariance is broken just as in a crystal, given that this phase is at the same time a superfluid it was not clear (at least to us) whether it was rigid. Here, we demonstrate by explicit calculation that this phase, which as we have discussed is plausibly the only form of quark matter that arises at densities reached within neutron star cores, is rigid indeed. Its shear modulus is parametrically of order $\Delta^{2} \mu^{2}$, which could have been guessed on dimensional grounds. The shear modulus is in no way suppressed relative to this natural scale, even though the crystalline color superconducting phase is superfluid.

Our paper is organized as follows. In Section II we describe the crystalline condensates which act as a background upon which the phonons act as 
small perturbations, fully specifying the CubeX and 2 Cube $45 z$ structures. In Section III, after fully specifying the NJL model that we shall use we introduce small displacements of a general diquark condensate which breaks translational symmetries. We write a general expression for the effective action describing these displacement fields, by integrating out the fermions in the system. The final result for the phonon effective action is given in Eq. (79). We relate the coefficients of the terms in the effective action involving the spatial derivatives of the displacement fields to the shear modulus in SubsectionIVA and then evaluate these coefficients for the CubeX and 2 Cube $45 z$ crystals in Subsections IV C andIVD respectively. We end with a discussion of our results and their consequences in Section V.

We have moved two relevant consistency checks to the Appendices to maintain continuity. In Appendix A we show explicitly that the displacement fields are massless to all orders in the gap parameter, as they must be by Goldstone's theorem. In Appendix B we evaluate the effective action for a simple "crystalline" structure involving just two flavors of quarks and pairing with only a single wave vector $\mathbf{q}$. In this case, the calculation can be done without making an expansion in the gap parameter, $\Delta$. We find that the results in the limit of small $\Delta$ are consistent with the Ginzburg-Landau calculation.

What are the observable signatures of the presence of quark matter in a crystalline color superconducting phase within the core of a neutron star? Prior to our work, the first steps toward calculating the cooling rate for such neutron stars were taken in Ref. 41]. Because the crystalline phases leave some quarks at their respective Fermi surfaces unpaired, it seems likely that their neutrino emissivity and heat capacity will be only quantitatively smaller than those of unpaired quark matter [42], not parametrically suppressed. This suggests that neutron stars with crystalline quark matter cores will cool by the direct URCA reactions, i.e. more rapidly than in standard cooling scenarios 43$]$. However, many other possible core compositions can open phase space for direct URCA reactions, making it unlikely that this will lead to a distinctive phenomenology [44]. In contrast, the enormous rigidity that we have identified makes the crystalline phases of quark matter unique among all forms of matter proposed as candidates for the composition of neutron star cores. This makes it particularly important to investigate its consequences. We close this
Introduction and close Section V of our paper with speculations about the consequences of a rigid quark matter core for pulsar glitch phenomenology, following the lead of Ref. 30].

A spinning neutron star observed as a pulsar gradually spins down as it loses rotational energy to electromagnetic radiation. But, every once in a while the angular velocity at the crust of the star is observed to increase suddenly in a dramatic event called a glitch. The standard explanation [45, 46, 47, 48, 49, 50, 51, 52, 53, 54, 55, 56, 57] for their occurrence requires the presence of a superfluid in some region of the star which also features a rigid array of spatial inhomogeneities which can pin the rotational vortices in the rotating superfluid. In the standard explanation of pulsar glitches, these conditions are met in the inner crust of a neutron star which features a crystalline array of positively charged nuclei bathed in a neutron superfluid (and a neutralizing fluid of electrons). The angular momentum of the rotating superfluid is proportional to the density of vortices, meaning that as the star as a whole slows the vortices "want" to move apart. As they are pinned to a rigid structure, they cannot. Hence, this superfluid component of the star is spinning faster than the rest of the star. After sufficient time, when the "tension" in the vortices in some region reaches a critical point, there is a sudden "avalanche" in which vortices unpin and rearrange, reducing the angular momentum of the superfluid. The rest of the star, including in particular the surface whose angular velocity is observed, speeds up. We see that this mechanism requires superfluidity coexisting with a structure that is rigid enough that it does not easily deform when vortices pinned to it seek to move, and an adequate pinning force which pins vortices to the rigid structure in the first place.

The crystalline color superconducting phases are superfluids: their condensates all spontaneously break the $U(1)_{B}$ symmetry corresponding to quark number. We shall always write the condensates as real. This choice of overall phase breaks $U(1)_{B}$, and gradients in this phase correspond to supercurrents. And yet, as we show at length in this paper, crystalline color superconductors are rigid solids with large shear moduli. Supersolids are another example of rigid superfluids [37, 38, 39], and on this basis alone one might consider referring to crystalline color superconducting phases as supersolid. However, supersolids are rigid by virtue of the presence of a conventional lattice of atoms whereas in a crys- 
talline color superconductor individual quarks are not fixed in place in a rigid structure - it is the crystalline pattern of the diquark condensate that is rigid, exhibiting a nonzero shear modulus. This makes crystalline color superconductors sufficiently different from supersolids that we shall refrain from borrowing the term.

Although our focus in this paper will be on the rigidity of crystalline color superconductors, it is worth pausing to consider how a condensate that vanishes at nodal planes can nevertheless carry a supercurrent. In an ordinary superfluid with spatially homogeneous condensate $\phi$, the ground state (with no current) can be taken to have $\phi$ real and the excited state with $\phi=\rho \exp i \theta(\mathbf{r})$ carries a current proportional to $\rho^{2} \nabla \theta$. If we now consider the simple example of a crystalline color superconductor with a diquark condensate of the form

$$
\Delta(\mathbf{r})=\Delta \cos (2 q z)
$$

this can carry current in the $x$ - and $y$-directions in the usual fashion but how can a current in the $z$ direction flow through the nodal planes at which $|\Delta(\mathbf{r})|$ vanishes? The answer is that in adddition to making the condensate complex, with a spatially varying phase, the presence of a current must deform the magnitude of the condensate slightly so as to make it nonvanishing at the location of the (former) nodal planes. A deformation that has all the appropriate properties is

$$
\Delta(\mathbf{r})=\Delta(\cos (2 q z)+i \epsilon \sin (2 q z)) .
$$

Note that if we write this condensate as $\Delta(\mathbf{r})=$ $\rho(z) \exp i \theta(z)$ it satisfies $\rho^{2} d \theta / d z=2 \epsilon q \Delta^{2}$, meaning that the current is $z$-independent as required by current conservation.) The deformation of the condensate is small for small $\epsilon$ as is the current, which is proportional to $\epsilon$. At the location of the former nodal planes, the magnitude of the condensate is also proportional to $\epsilon$. We shall leave the determination of whether (3) is the lowest energy deformation of the condensate (2) with current $2 \epsilon q \Delta^{2}$ to future work; the correct current carrying state must be similar, even if it does not turn out to be given precisely by (3). We shall also defer the extension of this analysis to the realistic, and more complicated, CubeX and 2 Cube $45 \mathrm{z}$ crystal structures. And, finally, we shall leave the analysis of currents flowing in circles in crystalline condensates - i.e. the construction of vortices - to future work.
It is reasonable to expect that the superfluid vortices that will result when crystalline color superconducting phases are rotated will have lower free energy if they are centered along intersections of the nodal planes of the underlying crystal structure, i.e. along lines along which the condensate already vanishes even in the absence of a rotational vortex. By virtue of being simultaneously superfluids and rigid solids, then, the crystalline phases of quark matter provide all the necessary conditions to be the locus in which (some) pulsar glitches originate. The shear modulus, which we calculate, is larger that that of a conventional neutron star crust by a factor of 20 to 1000, meaning that the rigidity of crystalline quark matter within a neutron star core is more than sufficient for glitches to originate therein. In Section $\mathrm{V}$ we also provide a crude estimate of the pinning force on vortices within crystalline color superconducting quark matter and find that it is comparable to the corresponding value for neutron vortices within a neutron star crust. Together, our calculation of the shear modulus and estimate of the pinning force make the core glitch scenario worthy of quantitative investigation. The central questions that remain to be addressed are the explicit construction of vortices in the crystalline phase and the calculation of their pinning force, as well as the timescale over which sudden changes in the angular momentum of the core are communicated to the (observed) surface, presumably either via the common electron fluid or via magnetic stresses.

\section{THREE FLAVOR CRYSTALLINE COLOR SUPERCONDUCTIVITY}

\section{A. Neutral unpaired quark matter}

We shall consider quark matter containing massless $u$ and $d$ quarks and $s$ quarks with an effective mass $M_{s}$. The Lagrangian density describing this system in the absence of interactions is given by

$$
\mathcal{L}_{0}=\bar{\psi}_{i \alpha}\left(i \not \partial_{i j}^{\alpha \beta}-M_{i j}^{\alpha \beta}+\mu_{i j}^{\alpha \beta} \gamma_{0}\right) \psi_{\beta j}
$$

where $i, j=1,2,3$ are flavor indices and $\alpha, \beta=$ $1,2,3$ are color indices and we have suppressed the Dirac indices. The mass matrix is given by $M_{i j}^{\alpha \beta}=$ $\delta^{\alpha \beta} \operatorname{diag}\left(0,0, M_{s}\right)_{i j}$, whereas $\partial_{i j}^{\alpha \beta}=\delta^{\alpha \beta} \delta_{i j} \partial$ and the 
quark chemical potential matrix is given by

$\mu_{i j}^{\alpha \beta}=\left(\mu \delta_{i j}-\mu_{e} Q_{i j}\right) \delta^{\alpha \beta}+\delta_{i j}\left(\mu_{3} T_{3}^{\alpha \beta}+\frac{2}{\sqrt{3}} \mu_{8} T_{8}^{\alpha \beta}\right)$,

with $Q_{i j}=\operatorname{diag}(2 / 3,-1 / 3,-1 / 3)_{i j}$ the quark electric-charge matrix and $T_{3}$ and $T_{8}$ the Gell-Mann matrices in color space. $\mu$ is the quark number chemical potential and if quark matter exists in neutron stars, we expect $\mu$ to be in the range $350-500 \mathrm{MeV}$.

In $\mathrm{QCD}, \mu_{e}, \mu_{3}$ and $\mu_{8}$ are the zeroth components of electromagnetic and color gauge fields, and the gauge field dynamics ensure that they take on values such that the matter is neutral [5, 58], satisfying

$$
\frac{\partial \Omega}{\partial \mu_{e}}=\frac{\partial \Omega}{\partial \mu_{3}}=\frac{\partial \Omega}{\partial \mu_{8}}=0
$$

with $\Omega$ the free energy density of the system. In the present paper we shall employ an NJL model with four-fermion interactions and no gauge fields. We introduce $\mu_{e}, \mu_{3}$ and $\mu_{8}$ by hand, and choose them to satisfy the neutrality constraints (6). The assumption of weak equilibrium is built into the calculation via the fact that for a given color $\alpha$ the chemical potential of the $d$ quark is equal the chemical potential of the $s$ quark which is equal to the sum of the chemical potential of the $u$ quark and $\mu_{e}$.

Let us consider now the effect of a nonvanishing strange quark mass $M_{s}$. Suppose we start by setting the gauge chemical potentials to zero. Then, in weak equilibrium a nonzero $M_{s}$ implies that there are fewer $s$ quarks in the system than $u$ and $d$ quarks, and hence the system is positively charged. To restore electrical neutrality, a positive $\mu_{e}$ is required which tends to reduce the number of up quarks relative to the number of down and strange quarks. In the absence of pairing, color neutrality is obtained with $\mu_{3}=\mu_{8}=0$.

To lowest order in $M_{s}^{2} / \mu^{2}$, the effect of a nonzero strange quark mass can be taken into account by treating the strange quark as massless, but with a chemical potential that is lowered by $M_{s}^{2} /(2 \mu)$ from $\mu+\left(\mu_{e} / 3\right)$. Indeed we can expand the Fermi momentum of the strange quark as

$$
\begin{aligned}
p_{F}^{s}=\sqrt{\left(\mu+\frac{\mu_{e}}{3}\right)^{2}-M_{s}^{2}} \approx & \left(\mu+\frac{\mu_{e}}{3}\right)-\frac{M_{s}^{2}}{2 \mu} \\
& +\mathcal{O}\left(\frac{M_{s}^{4}}{\mu^{3}}\right)
\end{aligned}
$$

and to this order electric neutrality requires that

$$
\begin{aligned}
& \mu_{e}=\frac{M_{s}^{2}}{4 \mu}, \text { yielding } \\
& p_{F}^{d}=\mu+\frac{M_{s}^{2}}{12 \mu}=p_{F}^{u}+\frac{M_{s}^{2}}{4 \mu} \\
& p_{F}^{u}=\mu-\frac{M_{s}^{2}}{6 \mu} \\
& p_{F}^{s}=\mu-\frac{5 M_{s}^{2}}{12 \mu}=p_{F}^{u}-\frac{M_{s}^{2}}{4 \mu} \\
& p_{F}^{e}=\frac{M_{s}^{2}}{4 \mu} .
\end{aligned}
$$

Now we need no longer to be careful about the distinction between $p_{F}$ 's and $\mu$ 's, as we can simply think of the three flavors of quarks as if they have chemical potentials

$$
\begin{aligned}
& \mu_{d}=\mu_{u}+2 \delta \mu_{3} \\
& \mu_{u}=p_{F}^{u} \\
& \mu_{s}=\mu_{u}-2 \delta \mu_{2},
\end{aligned}
$$

with

$$
\delta \mu_{3}=\delta \mu_{2}=\frac{M_{s}^{2}}{8 \mu} \equiv \delta \mu,
$$

and we can write the chemical potential matrix as

$$
\mu_{i j}^{\alpha \beta}=\delta^{\alpha \beta} \otimes \operatorname{diag}\left(\mu_{u}, \mu_{d}, \mu_{s}\right)_{i j} .
$$

The factor 2 in front of the $\delta \mu$ 's in Eq. (9) is taken to be consistent with the notation used in the analysis of crystalline superconductivity in a two flavor model 29], in which the two Fermi momenta were taken to be $\mu \pm \delta \mu$ meaning that they were separated by $2 \delta \mu$. In the three flavor case we have to contend with three combinations of pairs of Fermi surfaces and we define $2 \delta \mu_{I}=\left|\mu_{j}-\mu_{k}\right|$ where $j$ and $k$ are flavor indices different from each other and from $I$. The subscripts on the $\delta \mu$ 's in Eq. (9) are consistent with this definition. The most important driver of qualitative changes in the physics of quark matter as a function of decreasing $\mu$ is the increase in $\delta \mu=M_{s}^{2} /(8 \mu)$, the parameter which governs the splitting between the three Fermi momenta.

Finally, we note that the equality of $\delta \mu_{2}$ and $\delta \mu_{3}$ is only valid to leading order in $M_{s}^{2} / \mu^{2}$. At the next order, $\mu_{e}=M_{s}^{2} /(4 \mu)-M_{s}^{4} /\left(48 \mu^{3}\right)$ and hence $\delta \mu_{3}=$ $\mu_{e} / 2$ while $\delta \mu_{2}=\delta \mu_{3}+M_{s}^{4} /\left(16 \mu^{3}\right)$. The fact that $\delta \mu_{3}$ and $\delta \mu_{2}$ are close to, but not exactly, equal will be used in Appendix $\mathrm{A}$ in the explicit demonstration that the phonons are massless. The consequences of the fact that the splitting between the $u$ and $s$ Fermi surfaces is slightly larger than the splitting between the $u$ and $d$ Fermi surfaces were explored in Ref. [59]. 


\section{B. The crystalline condensate}

The description in Section $\llbracket A$ is valid in the absence of pairing between quarks. The interaction between quarks is attractive between two quarks that are antisymmetric in color. This induces the formation of Cooper pairs [3], and the ground state develops a nonzero diquark condensate which is predominantly antisymmetric in color indices. It is free-energetically favorable to have condensates that are antisymmetric in Dirac indices [2, 44, 5, 26] and consequently antisymmetric in flavor also. This implies that only quarks of different flavor form Cooper pairs.

The fact that quarks that like to pair have Fermi surfaces that are split in the absence of pairing motivates the possibility that quark matter may exist as a crystalline superconductor in at least some part of the parameter space 28, 29, 32]. This possibility was explored for three-flavor quark matter in Ref. [20] (and for simpler condensates in [18, 19]) where condensates of the form

$\left\langle\psi_{i \alpha}(x) \psi_{j \beta}(x)\right\rangle \propto C \gamma_{5} \sum_{I=1}^{3} \Delta_{I} \varepsilon_{I \alpha \beta} \varepsilon_{I i j} \sum_{\mathbf{q}_{I}^{a} \in\left\{\mathbf{q}_{I}\right\}} e^{2 i \mathbf{q}_{I}^{a} \cdot \mathbf{r}}$

were analyzed. As promised, the expression in Eq. (12) is antisymmetric in color, flavor and Dirac indices. For a given $I$ (which runs from 1 to 3), $\Delta_{I}$ represents the strength of the pairing between quarks whose color is not $I$ and whose flavor is not $I$. The periodic modulation in space of the $I$ 'th con- densate is defined by a set of momentum vectors $\left\{\mathbf{q}_{I}\right\}$. For example, pairing between $u$ and $d$ quarks occurs for $u d$-Cooper pairs with any momentum in the set $\left\{\mathbf{q}_{3}\right\}$. To shorten notation, we will often refer to the $\Delta_{I}$ collectively as $\Delta$ and will henceforth write $\mathbf{q}_{I}^{a} \in\left\{\mathbf{q}_{I}\right\}$ simply as $\mathbf{q}_{I}^{a}$.

The free energy, $\Omega$, and the gap parameters, $\Delta$, have been evaluated for many crystalline phases with condensates of the form (12) for varied crystal structures (i.e. varied $\left\{\mathbf{q}_{I}\right\}$ ) within the weak coupling and Ginzburg-Landau approximations $\Delta \ll \delta \mu \ll \mu[20]$.

In BCS-paired phases (like the CFL phase for example) quarks that pair have equal Fermi momenta even if in the absence of pairing their Fermi momenta would be split. This means that in such phases, the chemical potentials $\mu_{e}, \mu_{3}$ and $\mu_{8}$ take on different values than in the absence of pairing, with the differences being of order $M_{s}^{2} / \mu$. This rearrangement of Fermi surfaces exacts a free energy cost. The reason why crystalline phases can be more favorable is that they need pay no such cost: the quarks pair without rearranging Fermi surfaces; the gauge chemical potentials are as they would be in the absence of pairing, up to corrections that are proportional to $\Delta$ and thus negligible in the Ginzburg-Landau approximation. We therefore set $\mu_{e}=M_{s}^{2} /(4 \mu)$ and $\mu_{3}=\mu_{8}=0$ throughout, as in Ref. [20].

The free energy of a crystalline superconductor in the Ginzburg-Landau approximation relative to that for unpaired quark matter (i.e. the condensation energy) can be written as

$$
\begin{aligned}
\Omega_{\text {crystalline }}\left(\left\{\Delta_{I}\right\}\right)= & \frac{2 \mu^{2}}{\pi^{2}}\left[\sum_{I} P_{I} \alpha_{I} \Delta_{I}^{*} \Delta_{I}\right. \\
& +\frac{1}{2}\left(\sum_{I} \beta_{I}\left(\Delta_{I}^{*} \Delta_{I}\right)^{2}+\sum_{I>J} \beta_{I J} \Delta_{I}^{*} \Delta_{I} \Delta_{J}^{*} \Delta_{J}\right) \\
& \left.+\frac{1}{3}\left(\sum_{I} \gamma_{I}\left(\Delta_{I}^{*} \Delta_{I}\right)^{3}+\sum_{I \neq J} \gamma_{I J J} \Delta_{I}^{*} \Delta_{I} \Delta_{J}^{*} \Delta_{J} \Delta_{J}^{*} \Delta_{J}+\gamma_{123} \Delta_{1}^{*} \Delta_{1} \Delta_{2}^{*} \Delta_{2} \Delta_{3}^{*} \Delta_{3}\right)\right]+\mathcal{O}\left(\Delta^{8}\right),
\end{aligned}
$$

where $P_{I}$ is the number of momentum vectors in $\left\{\mathbf{q}_{I}\right\}$. The coefficients appearing in Eq. (13) have been calculated for several crystal structures in an NJL model [20], building upon previous analysis of 
the two-flavor case [30]. The quadratic coefficient, $\alpha_{I}$ depends only upon the magnitude of the momen- tum vectors in the set $\left\{\mathbf{q}_{I}\right\}$ and the Fermi surface splitting, $\delta \mu_{I}$, and is given by

$$
\alpha_{I}=\alpha\left(\mathbf{q}_{I}^{a}, \delta \mu_{I}\right)=\left[-1-\frac{1}{2} \log \left(\frac{2^{\frac{2}{3}} \Delta_{0}^{2}}{\left(\mathbf{q}_{I}^{a}\right)^{2}-\delta \mu_{I}^{2}}\right)+\frac{\delta \mu_{I}}{2\left|\mathbf{q}_{I}^{a}\right|} \log \left(\frac{\left|\mathbf{q}_{I}^{a}\right|+\delta \mu_{I}}{\left|\mathbf{q}_{I}^{a}\right|-\delta \mu_{I}}\right)\right]
$$

To find the most favorable magnitude of the vectors $\mathbf{q}_{I}^{a}$ we need to minimize the free energy with respect to $\left|\mathbf{q}_{I}^{a}\right|$ which at leading order is equivalent to minimizing $\alpha_{I}$ [30]. This fixes the ratio between the magnitude of the momentum vectors $\mathbf{q}_{I}^{a}$ and $\delta \mu_{I}$ to be the same for all the vectors,

$$
q_{I}=\left|\mathbf{q}_{I}^{a}\right|=\eta \delta \mu_{I} \quad \forall I, a,
$$

where $\eta$ satisfies the relation,

$$
1+\frac{1}{2 \eta} \log \left(\frac{\eta-1}{\eta+1}\right)=0
$$

and is given numerically by $\eta=1.1997$.

The higher order coefficients in Eq. (13) depend upon the relative directions $\left\{\hat{\mathbf{q}}_{I}\right\}$ of the vectors, and the task of finding favorable structures involves choosing polyhedra whose vertices are given by $\left\{\hat{\mathbf{q}}_{I}\right\}$, calculating $\Omega_{\text {crystalline }}$ for each choice, and finding those which have the lowest $\Omega_{\text {crystalline. For a given }}$ choice of crystal structure, the quartic coefficients in (13) (i.e. the $\beta$ 's) are dimensionless numbers times $\delta \mu^{2}$ and the sextic coefficients (i.e. the $\gamma$ 's) are dimensionless numbers times $\delta \mu^{4}$. This makes it clear that the control parameter for the GinzburgLandau approximation is $\Delta^{2} / \delta \mu^{2}=\Delta^{2} /\left(M_{s}^{2} / 8 \mu\right)^{2}$, which is of order $1 / 4$ for the most favorable crystal structures [20]. Because we shall only evaluate the phonon effective action and the shear modulus to order $\Delta^{2}$, we shall not need the values of the $\beta$ 's and $\gamma$ 's calculated in Ref. 20]; their role in the present calculation is only indirect, in the sense that they determine that the CubeX and $2 \mathrm{Cube} 45 \mathrm{z}$ structures are most favorable.

The free energy (13) simplifies upon taking $\Delta_{1}=$ $0 . \Delta_{1}$ can be neglected because it describes pairing between $s$ and $d$ quarks, whose Fermi momenta are separated by $\delta \mu_{1}$ which is twice $\delta \mu_{2}$ and $\delta \mu_{3}$.

The analysis of Ref. 20] yielded qualitative arguments that two crystal structures called CubeX and 2 Cube $45 \mathrm{z}$ should be most favorable, and explicit calculation of their free energy to order $\Delta^{6}$ in the Ginzburg-Landau expansion showed that they have comparable free energy to each other and have a lower (i.e. more favorable) free energy than all the other crystal structures considered. Furthermore, these two crystalline phases are favored with respect to unpaired quark matter and spatially uniform paired phases including the CFL and gCFL phases over the wide range of parameters (1). For example, taking $\Delta_{0}=25 \mathrm{MeV}$ and considering a fixed value of the strange quark mass $M_{s}=250 \mathrm{MeV}$, it turns out that one or other of the CubeX and 2 Cube $45 \mathrm{z}$ phases is favored for $\mu$ between $240 \mathrm{MeV}$ and $847 \mathrm{MeV}$, which more than covers the entire range of densities relevant for neutron star interiors. We shall now describe the CubeX and $2 \mathrm{Cube} 45 \mathrm{z}$ crystal structures.

\section{The CubeX and 2 Cube $45 z$ structures}

The CubeX crystal structure is specified by two sets of unit vectors, $\left\{\hat{\mathbf{q}}_{2}\right\}$ and $\left\{\hat{\mathbf{q}}_{3}\right\}$ depicted in the left panel of Fig. 1. Taken together, the two sets of vectors point towards the eight vertices of a cube. The four vectors in $\left\{\hat{\mathbf{q}}_{2}\right\}$ all lie in a plane and point towards the vertices of a diagonal rectangle of the cube, while the four vectors $\left\{\hat{\mathbf{q}}_{3}\right\}$ form the complementary rectangle. We will use a coordinate system such that $\left\{\hat{\mathbf{q}}_{2}\right\}$ is given by $\{(1 / \sqrt{3})( \pm \sqrt{2}, 0, \pm 1)\}$ (the four combinations of \pm giving the four vectors in $\left.\left\{\hat{\mathbf{q}}_{2}\right\}\right)$ and $\left\{\hat{\mathbf{q}}_{3}\right\}$ is given by $\{(1 / \sqrt{3})(0, \pm \sqrt{2}, \pm 1)\}$.

The 2Cube $45 \mathrm{z}$ crystal structure is specified by two sets of unit vectors, $\left\{\hat{\mathbf{q}}_{2}\right\}$ and $\left\{\hat{\mathbf{q}}_{3}\right\}$ depicted in the right panel of Fig. 1. The two sets $\left\{\hat{\mathbf{q}}_{2}\right\}$ and $\left\{\hat{\mathbf{q}}_{3}\right\}$ each contains eight vectors that point towards the vertices of a cube. The cubes specified by $\left\{\hat{\mathbf{q}}_{2}\right\}$ and $\left\{\hat{\mathbf{q}}_{3}\right\}$ are rotated relative to each other by an angle $45^{\circ}$ about one of their $C_{4}$ sym- 

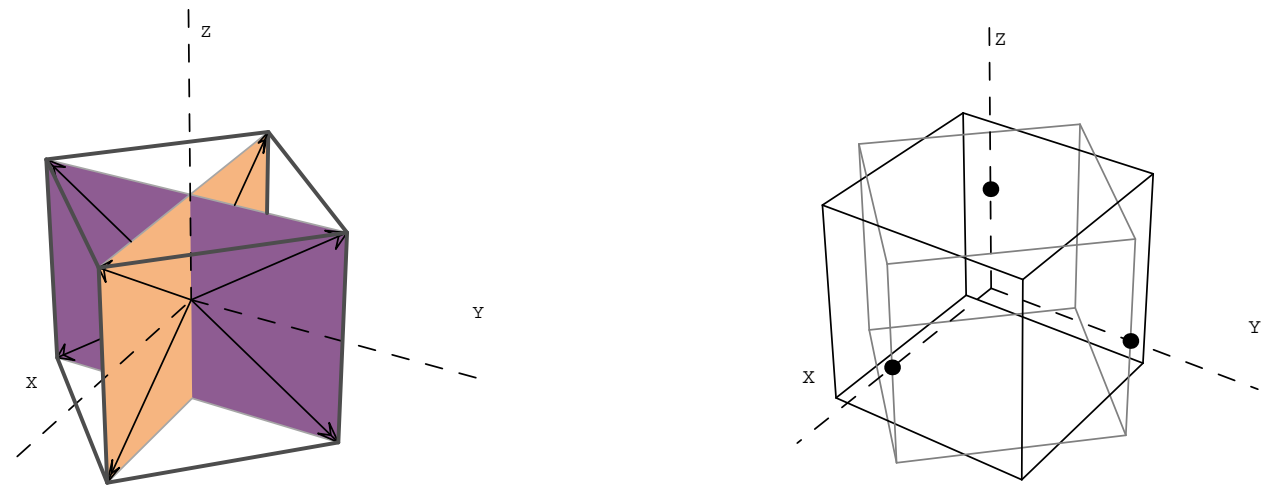

FIG. 1: (Color online) Left panel: The momentum vectors forming the CubeX crystal structure. This structure consists of eight vectors that belong to two sets $\left\{\hat{\mathbf{q}}_{2}\right\}$ and $\left\{\hat{\mathbf{q}}_{3}\right\}$ which are shown as vectors which start from the origin. The four vectors in $\left\{\hat{\mathbf{q}}_{2}\right\}$ are given by $(1 / \sqrt{3})( \pm \sqrt{2}, 0, \pm 1)$ and point toward the vertices of the light shaded rectangle (pink online) that lies in the $x-z$ plane. The four vectors in $\left\{\hat{\mathbf{q}}_{3}\right\}$ are given by $(1 / \sqrt{3})(0, \pm \sqrt{2}, \pm 1)$ and point toward the vertices of the dark shaded rectangle (purple online) that lies in the $y-z$ plane. Taken together the two sets of vectors point towards the eight vertices of the light gray colored cube (only the edges are shown as light gray segments). Right panel: The end points of the vectors forming the 2 Cube $45 \mathrm{z}$ crystal structure. This structure consists of sixteen vectors that belong to two sets $\left\{\hat{\mathbf{q}}_{2}\right\}$ and $\left\{\hat{\mathbf{q}}_{3}\right\}$. The eight elements of the set $\left\{\hat{\mathbf{q}}_{2}\right\}$ point towards the vertices of the black cube (only the edges are shown), and are given by $(1 / \sqrt{3})( \pm 1, \pm 1, \pm 1)$. The eight elements of the set $\left\{\hat{\mathbf{q}}_{3}\right\}$ point towards the vertices of the light gray cube, and are given by $\{(1 / \sqrt{3})( \pm \sqrt{2}, 0, \pm 1)\} \cup\{(1 / \sqrt{3})(0, \pm \sqrt{2}, \pm 1)\}$. The three dots denote the points where the axes meet the light gray cube, to clarify the orientation of the axes.

metry axis, passing through their common center. We will orient the coordinate axes such that $\left\{\hat{\mathbf{q}}_{2}\right\}$ is given by $\{(1 / \sqrt{3})( \pm 1, \pm 1, \pm 1)\}$ and $\left\{\hat{\mathbf{q}}_{3}\right\}$ by $\{(1 / \sqrt{3})( \pm \sqrt{2}, 0, \pm 1)\} \cup\{(1 / \sqrt{3})(0, \pm \sqrt{2}, \pm 1)\}$, which corresponds to a relative rotation by $45^{\circ}$ about the $\hat{z}$ axis.

The lattice spacing for the face-centered cubic crystal structure is [20, 30]

$$
a=\frac{\sqrt{3} \pi}{q}=\frac{4.536}{\delta \mu}=\frac{36.28 \mu}{M_{s}^{2}}
$$

For example, with $M_{s}^{2} / \mu=100,150,200 \mathrm{MeV}$ the lattice spacing is $a=72,48,36 \mathrm{fm}$. The spacing between nodal planes is $a / 2$.

\section{THE PHONON EFFECTIVE ACTION}

In this Section, we present our calculation of the effective action for the phonons in crystalline color superconducting phases of quark matter. In Subsection A we describe the NJL model which we use. In Subsections B and $\mathrm{C}$ we introduce the phonon field and integrate the fermions out, yielding a formal expression for the phonon effective action. In Subsections D and E we introduce the Ginzburg-Landau approximation and evaluate the phonon effective action to order $\Delta^{2}$. 


\section{A. NJL model and mean field approximation}

As described in Ref. [20], we work in an NJL model in which the quarks interact via a pointlike four-fermion interaction with the quantum numbers of single gluon exchange, in the mean field approximation. By this we mean that we add the local interaction term

$$
\mathcal{L}_{\text {interaction }}=\frac{3}{8} G(\bar{\psi} \Gamma \psi)(\bar{\psi} \Gamma \psi)
$$

to the Lagrangian density (4), and treat it in the mean field approximation as we describe below. Here, we have suppressed the color, flavor and Dirac indices; the full expression for the vertex factor is $\Gamma_{\alpha i, \beta j}^{A \nu}=\gamma^{\nu}\left(T^{A}\right)_{\alpha \beta} \delta_{i j}$, where the $T^{A}$ are the color Gell-Mann matrices. To regulate the ultraviolet behavior of the loop integrals, we introduce a cutoff $\Lambda$ which restricts the momentum integrals to a shell around the Fermi sphere: $\mu-\Lambda<|\vec{p}|<\mu+\Lambda$.

The free energy $\Omega$ and the gap parameter $\Delta$ for a crystalline superconductor in the weak coupling and Ginzburg-Landau approximations depend upon the NJL coupling $G$ and the cutoff $\Lambda$ only via $\Delta_{0}$, the gap parameter for the CFL phase with no Fermi surface splitting, given by

$$
\Delta_{0}=2^{\frac{2}{3}} \Lambda \exp \left[-\frac{\pi^{2}}{2 \mu^{2} G}\right] .
$$

Model calculations suggest that $\Delta_{0}$ should lie be- tween $10 \mathrm{MeV}$ and $100 \mathrm{MeV}$ [2]. In the weakcoupling approximation, $\Delta_{0} \ll \mu$ and it is possible to choose $\Lambda$ such that $\Lambda \gg \Delta_{0}$ while at the same time $\Lambda \ll \mu$.

We will see that the explicit dependence on $G$ disappears completely in our calculations and our results depend on the overall strength of the interactions only via the values of the gap parameters $\Delta_{I}$, which in turn depend on $G$ and $\Lambda$ only via the combination $\Delta_{0}$ [20]. It will nevertheless be useful to keep the cutoff $\Lambda$ in our loop integrals to see explicitly that all the pieces involved are finite. The final expressions for the coefficients of the terms that appear in the phonon effective action are ultraviolet safe, meaning, they are finite and $\Lambda$-independent as one takes $\Lambda \rightarrow \infty$. The weak-coupling approximation $\Delta_{0} \ll \mu$ is crucial to this result, as this allows one to take $\Lambda \gg \Delta_{0}$ while keeping $\Lambda \ll \mu$. Physically, the weak-coupling assumption means that the pairing is dominated by modes near the Fermi surfaces which means that the gaps, condensation energies and phonon effective action for the crystalline phases are independent of the cutoff in the NJL model as long as they are expressed in terms of the CFL gap $\Delta_{0}$ : if the cutoff is changed with the NJL coupling adjusted so that $\Delta_{0}$ stays fixed, the properties of the crystalline phases also stay fixed.

In the mean field approximation, the interaction Lagrangian (18) takes on the form

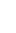

$$
\mathcal{L}_{\text {interaction }}=\frac{1}{2} \bar{\psi} \Delta(\mathbf{r}) \bar{\psi}^{T}+\frac{1}{2} \psi^{T} \bar{\Delta}(\mathbf{r}) \psi-\frac{3}{8} G \operatorname{tr}\left(\Gamma^{T}\left\langle\bar{\psi}^{T} \bar{\psi}\right\rangle \Gamma\right)\left\langle\psi \psi^{T}\right\rangle,
$$

where, tr represents the trace over color, flavor and Dirac indices, and where $\Delta(x)$ is related to the diquark condensate by the relations,

$$
\begin{aligned}
\Delta(x) & =\frac{3}{4} G \Gamma\left\langle\psi \psi^{T}\right\rangle \Gamma^{T} \\
\bar{\Delta}(x) & =\frac{3}{4} G \Gamma^{T}\left\langle\bar{\psi}^{T} \bar{\psi}\right\rangle \Gamma \\
& =\gamma^{0} \Delta^{\dagger}(x) \gamma^{0} .
\end{aligned}
$$

\section{B. Introduction of the phonon field}

We now consider the space- and time-dependent vibrations of the condensate, which will lead us to the effective Lagrangian for the phonon fields in the presence of a background condensate of the form (12). More precisely, we consider the condensate

$$
\Delta(\mathbf{r})=\Delta_{C F}(\mathbf{r}) \otimes C \gamma^{5}
$$


with

$$
\Delta_{C F}(\mathbf{r})_{\alpha i, \beta j}=\sum_{I=1}^{3} \varepsilon_{I \alpha \beta} \varepsilon_{I i j} \Delta_{I} \sum_{\mathbf{q}_{I}^{a}} e^{2 i \mathbf{q}_{I}^{a} \cdot \mathbf{r}} .
$$

Sometimes we will write

$$
\Delta_{C F}(\mathbf{r})=\sum_{I=1}^{3} \varepsilon_{I \alpha \beta} \varepsilon_{I i j} \Delta_{I}(\mathbf{r})
$$

with

$$
\Delta_{I}(\mathbf{r}) \equiv \Delta_{I} \sum_{\mathbf{q}_{I}^{a}} e^{2 i \mathbf{q}_{I}^{a} \cdot \mathbf{r}} .
$$

Note that $I=1,2,3$ correspond to $\langle d s\rangle,\langle u s\rangle$ and $\langle u d\rangle$ condensates, respectively. When we evaluate the phonon effective Lagrangian for the CubeX and 2 Cube $45 z$ crystals explicitly in Section IV, we will set $\Delta_{1}=0$ and $I$ will then run over 2 and 3 only. The condensate (23) breaks translation invariance spontaneously and we therefore expect Goldstone bosons corresponding to the broken symmetries, namely phonons. Phonons are small position and time dependent displacements of the condensate and, since the three condensates in (24) can oscillate independently, we expect there to be three sets of displacement fields $\mathbf{u}_{I}(x)$. In the presence of the phonons, then,

$$
\Delta_{I}(\mathbf{r}) \rightarrow \Delta_{I}^{u}(x)=\Delta_{I}\left(\mathbf{r}-\mathbf{u}_{I}(x)\right),
$$

and we will denote the corresponding quantities appearing on the left-hand sides of (24) and (22) as $\Delta_{C F}^{u}(x)$ and $\Delta^{u}(x)$ respectively, i.e.

$$
\Delta_{C F}^{u}(x)=\sum_{I=1}^{3} \varepsilon_{I \alpha \beta} \varepsilon_{I i j} \Delta_{I}^{u}(x)
$$

and

$$
\Delta^{u}(x)=\Delta_{C F}^{u}(x) \otimes C \gamma^{5} .
$$

(We apologize that we have denoted the displacement fields, and hence quantities like $\Delta^{u}$, by the letter $u$ which in other contexts, but not here, denotes up quarks.) In the mean field approximation, the full Lagrangian, given by the sum of (4) and (20), is quadratic in the fermion fields and can be written very simply upon introducing the two component Nambu Gorkov spinor

$$
\chi=\left(\begin{array}{c}
\psi \\
\bar{\psi}^{T}
\end{array}\right) \text { and hence } \bar{\chi}=\left(\bar{\psi} \psi^{T}\right)
$$

in terms of which

$$
\mathcal{L}=\frac{1}{2} \bar{\chi}\left(\begin{array}{cc}
i \not \partial+\not \mu & \Delta^{u}(x) \\
\bar{\Delta}^{u}(x) & (i \not \partial-\not \mu)^{T}
\end{array}\right) \chi+\frac{1}{16 G} \operatorname{tr}\left(\left(\bar{\Delta}^{u}\right)^{T} \Delta^{u}\right) .
$$

Here, $\mu \equiv \equiv \gamma_{0}$ and $\mu$ is the matrix we have defined in Eq. (11). In the next Sections, we shall also often use the notation $\mu_{i} \equiv \mu_{i} \gamma_{0}$, with $i=1,2,3$ corresponding to $u, d, s$ respectively. The last term in Eq. (30) comes from the last term in Eq. (20), which simplifies to $(1 /(16 G)) \operatorname{tr}\left(\left(\bar{\Delta}^{u}\right)^{T} \Delta^{u}\right)$ for condensates given by Eqs. (27) and (28).

\section{Integration over the $\chi$ fields}

The spacing between vortices in the vortex array in a rotating superfluid neutron star is many microns, and we will be interested in shear stresses exerted over lengths of order or longer than this length scale. This means that we need the effective action for phonon excitations with wavelengths of this order or longer. This length scale is many many orders of magnitude longer than the microscopic length scales that characterize the crystalline phase. For example, the lattice spacing is many tens of fm. This means that we need the effective action for phonons whose wavelength can be treated as infinite and whose energy can be treated as zero, certainly many many orders of magnitude smaller than $\Delta$.

The low energy quasiparticles in a crystalline color superconductor include the displacement fields $\mathbf{u}_{I}(x)$, which are massless because they are the Goldstone bosons of the broken translational symmetry. In addition, crystalline superconductors feature gapless fermionic modes, as we now explain. In the absence of pairing, quarks living at the Fermi surfaces can be excited without any cost in free energy; pairing in the crystalline phases yields gaps for quarks living in various ring-shaped bands around the Fermi surfaces, but leaves gapless fermionic modes at the boundaries of the pairing regions (loosely speaking, the remainder of the original Fermi surfaces other than the ring-shaped bands) [20, 29, 30, 32]. The low energy effective theory includes fermions in the vicinity of the surfaces in momentum space that bound the pairing regions, in addition to the phonons that are our primary interest in this paper.

To find the low energy effective action which describes the phonons and the gapless fermionic excitations we need to integrate out those fermion fields 
which have an energy larger than some infrared cutoff $\Lambda_{\mathrm{IR}}$. For the application of interest to us, $\Lambda_{\mathrm{IR}}$ should be the energy of phonons with micron wavelengths. If we were interested in thermal properties, $\Lambda_{\text {IR }}$ would be of order the temperature $T$. (Either of these energy scales is $\ll \Delta$, and by the end of this Subsection we will see that it is safe to set $\Lambda_{\mathrm{IR}}=0$.) In order to formally implement this procedure, we define

$$
\psi=\psi_{\rangle}+\psi_{\langle} \text {and hence, } \quad \bar{\psi}=\bar{\psi}_{\rangle}+\bar{\psi}_{\langle},
$$

where $\psi_{<}$and $\bar{\psi}_{<}$contain modes with energy in $\left[0, \Lambda_{\mathrm{IR}}\right]$ and $\psi_{\rangle}$and $\bar{\psi}_{\rangle}$those with energy in $\left[\Lambda_{\mathrm{IR}}, \infty\right]$. Note that the boundary in momentum space between the $\psi_{\rangle}$and $\psi_{<}$modes will be nontrivial surfaces that follow the boundaries of the pairing regions, where the gapless fermions are found. The corresponding decomposition for the Nambu Gorkov fields is,

$$
\chi=\chi_{\rangle}+\chi_{\langle} \text {and hence } \bar{\chi}=\bar{\chi}_{\rangle}+\bar{\chi}_{\langle},
$$

where $\chi_{\rangle}, \chi_{\langle}, \bar{\chi}_{\rangle}$and $\bar{\chi}_{\langle}$are defined analogously to Eq. (29). Carrying out the functional integral over the $\chi_{\rangle}$and $\left.\bar{\chi}\right\rangle$ fields will leave us with a low-energy effective action in terms of the $\mathbf{u}_{I}, \chi_{<}$and $\bar{\chi}_{<}$fields.

We begin with the path integral expression for the partition function,

$$
Z\left[\mathbf{u}, \chi_{\langle}, \bar{\chi}_{\curlywedge}\right]=\int \mathcal{D}\left[\chi_{\rangle}\right] \mathcal{D}\left[\bar{\chi}_{\rangle}\right] e^{i \int d^{4} x \mathcal{L}},
$$

where the action of the Lagrangian in Eq. (30) can be written in terms of the decomposed fields (32), as follows,

$$
\int d^{4} x \mathcal{L}=\int d^{4} x\left[\frac{1}{16 G} \operatorname{tr}\left(\left(\bar{\Delta}^{u}\right)^{T} \Delta^{u}\right)+\bar{\chi} S^{-1} \chi_{\rangle}+\bar{\chi}_{\langle} S^{-1} \chi_{\curlywedge}\right],
$$

where the cross terms $\bar{\chi}\rangle S^{-1} \chi_{\langle}$and $\bar{\chi}_{\langle} S^{-1} \chi_{\rangle}$do not appear because the integration over $x^{0}$ imposes energy conservation, and $\chi_{\rangle}$and $\chi_{<}$have support over different ranges of energy. The full inverse propagator, $S^{-1}$, in Eq. (34) is given by

$$
S^{-1}=\left(\begin{array}{cc}
i \not \partial+\not \mu & \Delta^{u}(x) \\
\bar{\Delta}^{u}(x) & (i \not \partial-\not \mu)^{T}
\end{array}\right) .
$$

Since the Lagrangian is quadratic in the $\chi$ and $\bar{\chi}$ fields, the standard result for fermionic functional integrals gives

$$
i \mathcal{S}\left[\mathbf{u}, \chi_{<}, \bar{\chi}_{\curlywedge}\right]=\log \left(Z\left[\mathbf{u}, \chi_{<}, \bar{\chi}_{\zeta}\right]\right)=i \int d^{4} x\left[\bar{\chi}_{\langle} S^{-1} \chi_{\curlywedge}+\frac{1}{16 G} \operatorname{tr}\left(\left(\bar{\Delta}^{u}\right)^{T} \Delta^{u}\right)\right]+\frac{1}{2} \operatorname{Tr}_{\mathrm{ng}} \log \left(S^{-1}\right)
$$

where $\mathcal{S}\left[\mathbf{u}, \chi_{<}, \bar{\chi}_{<}\right]$is the low energy effective action that we are after, at present still given at a rather formal level. Here, $\operatorname{Tr}_{n g}$ symbolizes the trace over the Nambu-Gorkov index, the trace over color, flavor, Dirac indices and the trace over a set of functions on space-time, with energies lying in $\left[-\infty,-\Lambda_{\mathrm{IR}}\right] \cup\left[\Lambda_{\mathrm{IR}}, \infty\right]$. The factor $\frac{1}{2}$ appears before $\operatorname{Tr}_{\text {ng }}$ because all the components of $\chi$ and $\bar{\chi}$ are not independent. The actual independent fields are $\psi$ and $\bar{\psi}$. As promised, the effective action is a function of the low energy quark fields, which appear in $\bar{\chi}_{\langle} S^{-1} \chi_{<}$, and the phonon fields, which appear implicitly via the dependence of $S^{-1}$ and $\Delta^{u}$ on $\mathbf{u}_{I}$.

We now concentrate on small displacements and hence drop all terms in the effective action of order $\left(\mathbf{u}_{I}\right)^{3}$ or higher. This is most simply done by looking 


$$
\begin{aligned}
\Delta_{C F}^{u}(x) & =\sum_{I=1}^{3} \varepsilon_{I \alpha \beta} \varepsilon_{I i j} \Delta_{I} \sum_{\mathbf{q}_{I}^{a}} e^{2 i \mathbf{q}_{I}^{a} \cdot\left(\mathbf{r}-\mathbf{u}_{I}(x)\right)}, \\
& =\sum_{I=1}^{3} \varepsilon_{I \alpha \beta} \varepsilon_{I i j} \Delta_{I} \sum_{\mathbf{q}_{I}^{a}} e^{2 i \mathbf{q}_{I}^{a} \cdot \mathbf{r}}\left(1-i \phi_{I}^{a}(x)-\frac{1}{2}\left(\phi_{I}^{a}(x)\right)^{2}\right)+\mathcal{O}(\phi(x))^{3},
\end{aligned}
$$

where we have defined

$$
2 \mathbf{q}_{I}^{a} \cdot \mathbf{u}_{I}(x)=\phi_{I}^{a}(x) .
$$

We will refer to both the $\mathbf{u}_{I}$ fields and $\phi_{I}^{a}$ fields as phonons, as we can write one in terms of the other.

We now argue that as far as the calculation of the shear modulus is concerned, we can look only at the part of the effective action that describes the phonons. The remainder of the effective Lagrangian, where the low energy quark fields appear, can be written as

$$
\bar{\chi}_{\langle} S^{-1} \chi_{<}=\mathcal{L}_{f}+\mathcal{L}_{f \phi}
$$

with

$$
\mathcal{L}_{f}=\bar{\chi}_{<}\left(\begin{array}{cc}
i \not \partial+\not \mu & \Delta(\mathbf{r}) \\
\bar{\Delta}(\mathbf{r}) & (i \not \partial-\not \mu)^{T}
\end{array}\right) \chi_{<}
$$

and

$$
\mathcal{L}_{f \phi}=\bar{\chi}_{\iota}\left(\begin{array}{cc}
0 & \Delta^{u}(x)-\Delta(\mathbf{r}) \\
\bar{\Delta}^{u}(x)-\bar{\Delta}(\mathbf{r}) & 0
\end{array}\right) \chi_{<} .
$$

We shall see in Section IVA that the shear modulus is related to the coefficient of

$$
\frac{\partial \phi}{\partial x^{u}} \frac{\partial \phi}{\partial x^{v}}
$$

in the Lagrangian, which makes it obvious that $\mathcal{L}_{f}$ does not contribute. The coefficient of (42) at the scale $\Lambda_{\mathrm{IR}}$ receives contributions from the $\chi_{\rangle}$and $\bar{\chi}\rangle$ fermions which have been integrated out. The phonons and low energy quarks (at an energy scale lower than $\Lambda_{I R}$ ) interact via the $\mathcal{L}_{f \phi}$ term in the Lagrangian. Formally, then, one has to solve consistently for the phonon propagator and the quark propagator, which are coupled. However, the effect of the phonon-fermion interactions on the self consistent calculation of the gap parameter using $\mathcal{L}_{f}$ is small, because the quark loops come with an additional factor of $\mu^{2}$, which is is much larger than $\left(\Lambda_{\mathrm{IR}}\right)^{2}$, and hence the quark propagator can be considered to be unaffected by the phonons. The phonon propagator, and hence the shear modulus, will depend on the phonon-fermion interactions, meaning that the phonon propagator and consequently the coefficient of (42) will run as $\Lambda_{\mathrm{IR}}$ is reduced. However, as long as $\Lambda_{\mathrm{IR}}$ is much smaller than $\Delta,|\mathbf{q}|$ and $\delta \mu$, the change in the value of the shear modulus from integrating out more fermions below the scale $\Lambda_{\mathrm{IR}}$ will be negligible compared to its value at $\Lambda_{\mathrm{IR}}$. This means that we can take $\Lambda_{\mathrm{IR}}=0$, integrating all of the fermions out from the system and obtaining an effective action for the phonons alone. This procedure is correct for the calculation of the shear modulus but would not be correct for, say, calculating the specific heat of the system, which is dominated by the gapless fermions not by the phonons. We have checked numerically that the difference between the shear modulus calculated with $\Lambda_{\mathrm{IR}}=0$ and that with a small but nonzero $\Lambda_{\mathrm{IR}}$ is negligible.

Finally, therefore, the effective action we are interested in depends only on the phonon fields, and is given by

$$
i \mathcal{S}[\mathbf{u}]=\log (Z[\mathbf{u}])=i \int d^{4} x\left[\frac{1}{16 G} \operatorname{tr}\left(\left(\bar{\Delta}^{u}\right)^{T} \Delta^{u}\right)\right]+\frac{1}{2} \operatorname{Tr}_{\mathrm{ng}} \log \left(S^{-1}\right)
$$


where now the $\operatorname{Tr}_{n g}$ includes a trace over functions in space-time containing all energy modes.

For the single plane wave "crystal" structure in which only one of the $\Delta_{I}$ is nonzero and $\left\{\mathbf{q}_{I}\right\}$ contains only a single wave vector [28, 29], we can invert the Nambu-Gorkov inverse propagator in the absence of phonons without expanding in $\Delta$, and can therefore obtain the effective action for the phonons up to second order in $\phi$, to all orders in $\Delta$. We shall do this exercise in Appendix B For the realistic crystal structures, CubeX and 2Cube45z, however, we cannot invert the full inverse propagator and we therefore proceed by making a Ginzburg-Landau expansion in $\Delta$.

\section{Ginzburg-Landau expansion}

In order to obtain the effective action for the phonon field we first separate the full inverse prop- agator, $S^{-1}$, defined in Eq. (35), into the free part, $S_{0}^{-1}$ and a part containing the condensate, $\Sigma$, as follows: $S^{-1}=S_{0}^{-1}+\Sigma$, where

$$
S_{0}^{-1}=\left(\begin{array}{cc}
i \not \partial+\not \mu & 0 \\
0 & (i \not \partial-\not L)^{T}
\end{array}\right)
$$

and

$$
\Sigma=\left(\begin{array}{cc}
0 & \Delta^{u}(\mathbf{r}) \\
\bar{\Delta}^{u}(\mathbf{r}) & 0
\end{array}\right)
$$

Then, we can expand the term $\log \left(S^{-1}\right)$ that appears on the right-hand side of Eq. (43) as

$$
\operatorname{Tr}_{\mathrm{ng}}\left(\log \left(S_{0}^{-1}+\Sigma\right)\right)=\operatorname{Tr}_{\mathrm{ng}}\left(\log S_{0}^{-1}\right)+\operatorname{Tr}_{\mathrm{ng}}\left(S_{0} \Sigma\right)-\frac{1}{2} \operatorname{Tr}_{\mathrm{ng}}\left(S_{0} \Sigma\right)^{2}+\ldots
$$

where $\operatorname{Tr}_{\mathrm{ng}}\left(\log S_{0}^{-1}\right)$ is related to the free energy of unpaired (Normal) quark matter $\Omega_{N}$ by

$$
\operatorname{Tr}_{\mathrm{ng}}\left(\log S_{0}^{-1}\right)=-i 2 \Omega_{N} \int d^{4} x=-i 2 V T \Omega_{N}
$$

with $V T$ the space-time volume. Since

$$
\left(S_{0} \Sigma\right)=\left(\begin{array}{cc}
0 & (i \not \partial+\not \mu)^{-1} \Delta^{u}(x) \\
\left((i \not \partial-\not \mu)^{T}\right)^{-1} \bar{\Delta}^{u}(x) & 0
\end{array}\right)
$$

only even powers of $\left(S_{0} \Sigma\right)$ contribute to the trace over Nambu Gorkov indices and we can write,

$$
\operatorname{Tr}_{\mathrm{ng}}\left(\log \left(S^{-1}\right)\right)=-i 2 \Omega_{N}(V T)-\sum_{n=1}^{\infty} \frac{1}{n} \operatorname{Tr}\left((i \not \partial+\not l)^{-1} \Delta^{u}(x)\left((i \not \partial-\not \iota)^{T}\right)^{-1} \bar{\Delta}^{u}(x)\right)^{n}
$$

where, the Tr on the right hand side is over Dirac, color, flavor and space-time, and we have used the cyclic property of the trace to equate the two contributions obtained from the trace over the Nambu Gorkov index. Finally, substituting (49) back in (43) and simplifying the Dirac structure of the operators using the $C \gamma^{5}$ Dirac structure of the condensate and the properties of the charge conjugation matrix $C$, namely $C\left(\gamma^{\mu}\right)^{T} C^{-1}=-\gamma^{\mu}$ and $C^{2}=-1$, we obtain 


$$
\mathcal{S}[\mathbf{u}]=-\frac{1}{4 G} \int d^{4} x \operatorname{tr}_{C F}\left(\left(\Delta_{C F}^{u}\right)^{\dagger} \Delta_{C F}^{u}\right)-\Omega_{N}(V T)-\frac{1}{2 i} \sum_{n=1}^{\infty} \frac{1}{n} \operatorname{Tr}\left((i \not \partial+\not)^{-1} \Delta_{C F}^{u}(x)(i \not \partial-\not)^{-1} \Delta_{C F}^{u \dagger}(x)\right)^{n}
$$

where the $\operatorname{trace}^{\operatorname{tr}_{C F}}$ is over color and flavor indices and where $\Delta_{C F}^{u}(x)$ depends on $\mathbf{u}_{I}(x)$ via Eqs. (37) and (38). Eq. (50) is the low energy effective action for the phonons, written as a Ginzburg-Landau expansion in $\Delta$. We will calculate the leading contribution to $\mathcal{S}[\mathbf{u}]$, namely that proportional to $\Delta^{2}$.

The first term on the right hand side of Eq. (50) does not have any derivatives acting on $\mathbf{u}_{I}$ and hence can only contribute to the mass of the phonon, which we know must be zero by Goldstone's theorem. In Appendix $\mathrm{A}$ we show explicitly that the $\mathbf{u}_{I}$ dependence in $\int d^{4} x \operatorname{tr}_{C F}\left(\left(\Delta_{C F}^{u}\right)^{\dagger} \Delta_{C F}^{u}\right)$ cancels out, and its value is given simply by

$$
\begin{aligned}
\frac{1}{4 G} \int d^{4} x \operatorname{tr}_{C F}\left(\left(\Delta_{C F}^{u}\right)^{\dagger} \Delta_{C F}^{u}\right) \\
=(V T) \frac{1}{G} \sum_{I}\left(\Delta_{I} \Delta_{I}^{*}\right) P_{I},
\end{aligned}
$$

where $P_{I}$ is the number of plane waves in $\left\{\mathbf{q}_{I}\right\}$.

We now proceed to evaluate the third term on the right hand side of Eq. (50) diagrammatically. We will expand the action $\mathcal{S}[\mathbf{u}]$ in Eq. (50) in powers of $\phi$ (or equivalently $\mathbf{u}_{I}$ ) up to second order in $\phi$ by using the Feynman rules described in Fig. 2 .

The lowest order term is independent of $\phi$. The sum of this term and of the term given in Eq. (51) has a simple interpretation. In the absence of phonons, and considering that the fermionic fields have been integrated out, the action in Eq. (50) turns out to be proportional to the free energy of the system. More specifically,

$$
\mathcal{S}[\mathbf{u}=0]=-(V T)\left(\Omega_{\text {crystalline }}+\Omega_{N}\right),
$$

where $\Omega_{\text {crvstalline }}$ is given as a Ginzburg-Landau series in $\Delta[20]$. Since (52) is independent of $\phi$ it does not affect the equations of motion of the phonons and we will simply drop it from our calculation.

We now consider the term that is linear in $\phi$. We will evaluate the leading term in the action proportional to $\Delta^{2}$, which we will call $\mathcal{S}^{\phi \Delta^{2}}$ and which is represented diagrammatically in Fig. 3. Both terms shown in Fig. 3 are proportional to the trace of $\varepsilon_{I \alpha \beta} \varepsilon_{J \alpha \beta} \varepsilon_{I i j} \varepsilon_{J i j}$, which is nonzero only if $I=J$ and therefore only terms proportional to $\Delta_{I}^{*} \Delta_{I}$ are present. We could have anticipated this result from the symmetries of the problem. The Lagrangian conserves particle number for every flavor of quarks, which corresponds to symmetry under independent global phase rotations of quark fields of the three flavors, meaning independent phase rotations of the three $\Delta_{I}$. The effective action should be invariant under these rotations and hence $\Delta_{I}$ can only occur in the combination $\Delta_{I}^{*} \Delta_{I}$. (Although the condensate spontaneously breaks them, the requirement is that the Lagrangian has these symmetries.) Then, the sum of the diagrams in Fig. 3, which corresponds to the contribution to the action linear in the phonon field and second order in $\Delta$, is given by

$$
\begin{aligned}
\mathcal{S}^{\phi \Delta^{2}}= & \sum_{I} \Delta_{I}^{*} \Delta_{I} \sum_{\substack{j \neq k \\
\neq \neq}} \sum_{\mathbf{q}_{I}^{a} \mathbf{q}_{I}^{b}} \int \frac{d^{4} k}{(2 \pi)^{4}} \int \frac{d^{4} p}{(2 \pi)^{4}}(2 \pi)^{4} \delta^{(4)}\left(2 \mathbf{q}_{I}^{a}-2 \mathbf{q}_{I}^{b}+k\right) \\
& \operatorname{tr}\left[\frac{\phi_{I}^{a}(k)-\phi_{I}^{b}(k)}{\left(\not p+2 \phi_{I}^{b}+\not k+\not \mu_{j}\right)\left(\not p-2\left(\phi_{I}^{a}-\phi_{I}^{b}\right)+\not k-\not \mu_{k}\right)}\right],
\end{aligned}
$$

where $k$ is the four momentum of the phonon field and the trace is over Dirac indices. The Dirac delta on the right-hand side ensures momentum conserva- 

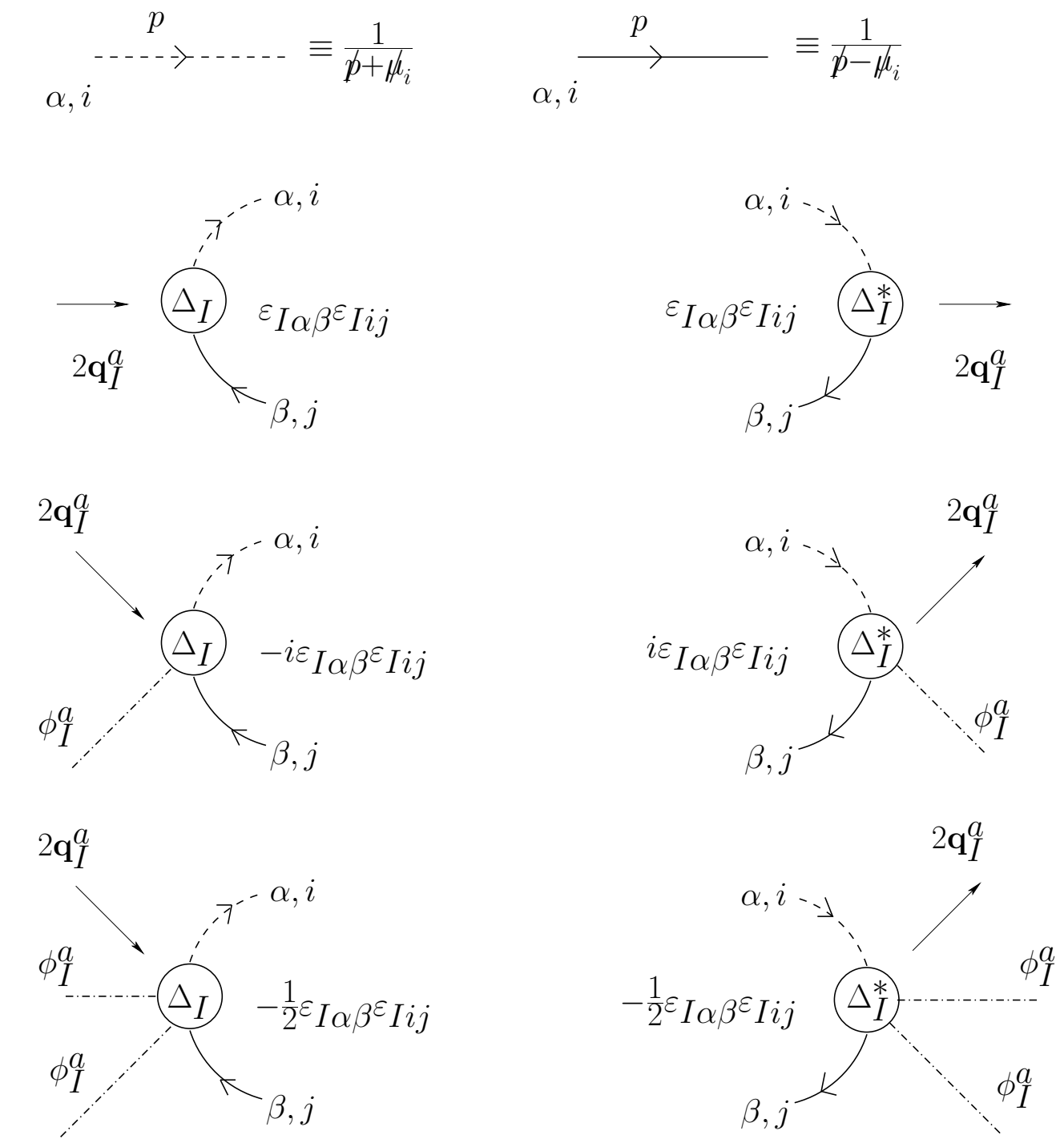

FIG. 2: Propagators and interaction vertices for the Lagrangian up to order $\phi^{2}$. The dashed lines represent propagating quarks, and the solid lines represent propagating quark holes. The dot-dash lines represent external phonons. The subscript on $\mu$ is the index of the flavor which is propagating, and determines the value of the chemical potential that appears in the propagator. The $\Delta_{I}$ vertex comes along with a momentum insertion $2 \mathbf{q}_{I}^{a}$ and a vertex factor $\varepsilon_{I \alpha \beta} \varepsilon_{I i j}$. Similarly, $\Delta_{I}^{*}$ comes with a momentum insertion $-2 \mathbf{q}_{I}^{a}$ and the same vertex factor.

tion,

$$
2 \mathbf{q}_{I}^{a}-2 \mathbf{q}_{I}^{b}+k=0,
$$

meaning that the net momentum added to the loop is zero. But since we are looking at the low energy effective theory, we can take $k$ much smaller than the momentum vectors $\mathbf{q}_{I}$ and therefore Eq. (54) can be satisfied only if $k=0$ and $\mathbf{q}_{I}^{a}=\mathbf{q}_{I}^{b}$, which means that $a=b$. Using this result, we find that (53) vanishes:

$$
\mathcal{S}^{\phi \Delta^{2}}=0 .
$$

That is, the term linear in $\phi$ is absent to order $\Delta^{2}$. One can similarly argue that it is absent to all orders in $\Delta$. 

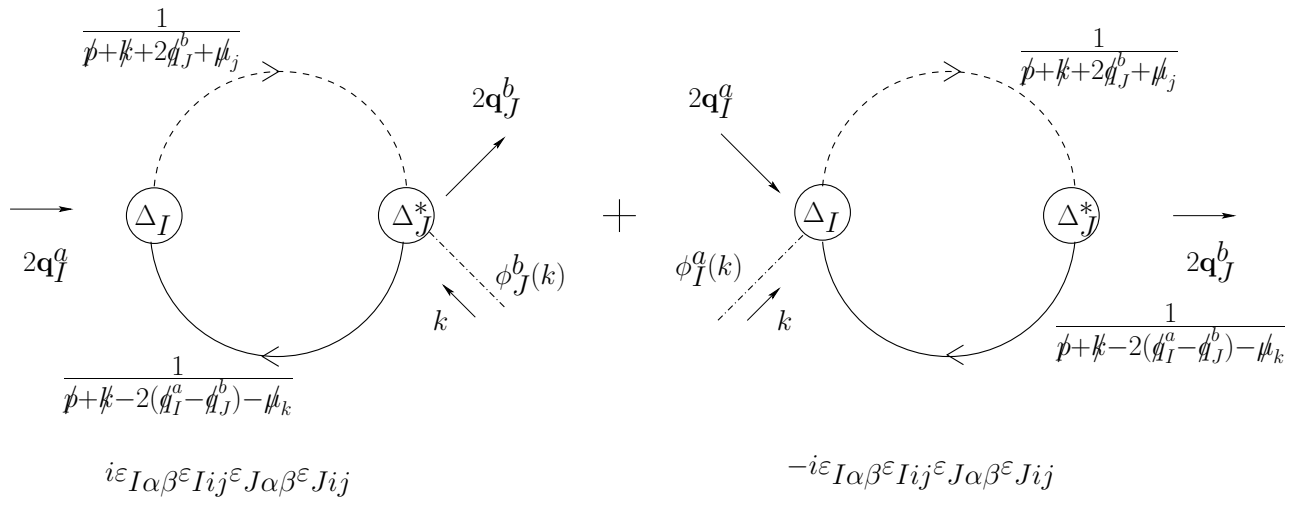

FIG. 3: Diagrams that contribute to order $\phi \Delta^{2}$. The dashed lines represent propagating quarks, and the solid lines represent propagating quark holes. The interaction vertices have been defined in Fig. 2 and the color-flavor structure is also indicated. Note that the trace over the color-flavor epsilon tensors, $\varepsilon_{I \alpha \beta} \varepsilon_{J \alpha \beta} \varepsilon_{I i j} \varepsilon_{I i j}$ forces $I=J$ and momentum conservation implies $\mathbf{q}_{I}^{a}=\mathbf{q}_{I}^{b}$, as well as $k=0$. The two contributions are then equal in magnitude and opposite in sign, and hence cancel.

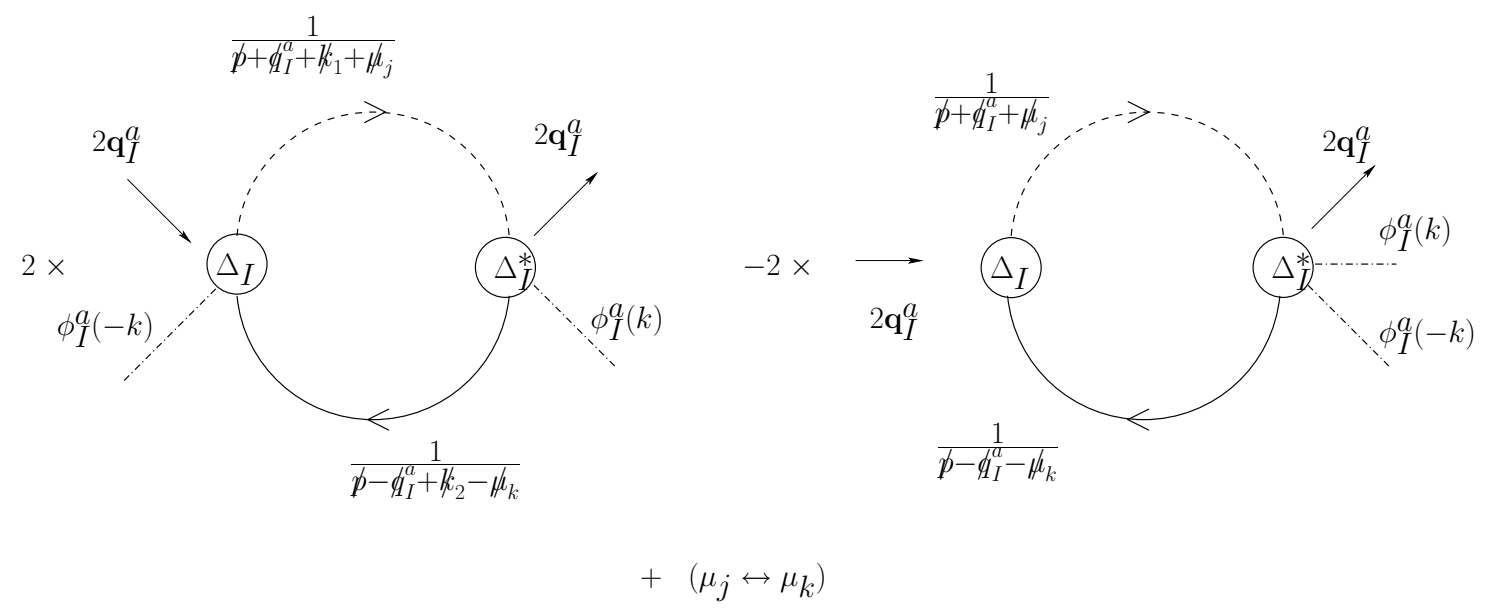

FIG. 4: Diagrams that contribute to order $\phi^{2} \Delta^{2}$. In drawing the diagrams, we have used the fact that the trace of the color-flavor tensor forces $I=J$. We have also used the fact that momentum conservation requires that the net momenta added by the condensate and the phonons are separately zero. In the first diagram, momentum conservation at the $\Delta_{I}^{*}$ vertex imposes $k_{2}-k_{1}=k$.

Now we consider the terms of order $\phi^{2}$, which give the first nontrivial contribution to the phonon effective action. We will evaluate these terms to order $\Delta^{2}$ and we will call the corresponding contribution to the action $\mathcal{S}^{\phi^{2} \Delta^{2}}$. The terms contributing to $\mathcal{S}^{\phi^{2} \Delta^{2}}$ arise from the diagrams given in Fig. [ and give

$$
\mathcal{S}^{\phi^{2} \Delta^{2}}=\sum_{I} \sum_{\mathbf{q}_{I}^{a}} \int \frac{d^{4} k}{(2 \pi)^{4}} \phi_{I}^{a}(k) \phi_{I}^{a}(-k) \Delta_{I}^{*} \Delta_{I} \mathcal{P}_{I}^{a}(k),
$$


where $k=k_{2}-k_{1}$ is the four momentum of the phonon and

$$
\begin{gathered}
\mathcal{P}_{I}^{a}(k)=i \sum_{\substack{j \neq k \\
\neq I}} \int \frac{d^{4} p}{(2 \pi)^{4}} \operatorname{tr}\left[\frac{1}{\left(\not p+\not \phi_{I}^{a}+\not k_{1}+\not \mu_{j}\right)\left(\not p-\not \phi_{I}^{a}+\not k_{2}-\not k_{k}\right)}\right. \\
\left.-\frac{1}{\left(\not p+\not \phi_{I}^{a}+\not \mu_{j}\right)\left(\not p-\not q_{I}^{a}-\not \mu_{k}\right)}\right],
\end{gathered}
$$

where the trace is over Dirac indices. In the next Subsection we evaluate Eq. (57). The reader not interested in the details of our calculation will find in Eq. (78) the final expression for the effective action $\mathcal{S}^{\phi^{2} \Delta^{2}}$.

\section{E. Evaluation of $\mathcal{S}^{\phi^{2} \Delta^{2}}$}

We turn now to the evaluation of $\mathcal{P}_{I}^{a}(k)$ of Eq. (57) and hence, via (56), the leading nontrivial contribution to the phonon low energy effective action, $\mathcal{S}^{\phi^{2} \Delta^{2}}$.

To begin, if we set $k_{1}=k_{2}=0$ in (57), implying that $k=0$, we see that $\mathcal{P}_{I}^{a}(0)=0$. In this way, we see explicitly that the phonons are massless to order $\Delta^{2}$. As mentioned before, we are interested in the low energy, long wavelength phonons. We therefore expand $\mathcal{P}_{I}^{a}(k)$ in powers of $k$ and drop terms of order $k^{3}$ and higher.

We are working in the limit in which $\delta \mu, q=|\mathbf{q}|=$ $\eta \delta \mu$ and $\Delta$ are all much smaller than $\mu$. $(\Delta \ll \mu$ follows from the weak coupling approximation and $\delta \mu \ll \mu$ follows from requiring $M_{s}^{2} \ll \mu^{2}$. The Ginzburg-Landau approximation, which is the further requirement that $\Delta^{2} \ll \delta \mu^{2}$, is not required in the derivation of the simplifications of Eq. (57) that follow.) This means that we can choose the ultraviolet cutoff $\Lambda$ such that $\delta \mu, q, \Delta \ll \Lambda \ll \mu$. Since the largest contribution to the integrals comes from the region close to the Fermi surfaces and since $\Lambda \ll \mu$, the integration measure in Eq. (57) can be approximated as follows:

$$
i \int \frac{d^{4} p}{(2 \pi)^{4}} \approx \frac{i \mu^{2}}{2 \pi^{2}} \int_{-\infty}^{+\infty} \frac{d p^{0}}{2 \pi} \int_{-\Lambda}^{\Lambda} d s \int \frac{d \hat{\mathbf{v}}}{4 \pi}
$$

where $\hat{\mathbf{v}}$ is the unit velocity vector in the direction of the spatial momentum vector, $\hat{\mathbf{v}}=\mathbf{p} /|\mathbf{p}|$, and $\int d \hat{\mathbf{v}}$ represents the integral over solid angle covering the Fermi surface. The residual momentum $s$ is defined by the relation $s \equiv|\mathbf{p}|-\bar{\mu}$, where $\bar{\mu}$ is an energy scale lying close to the quark Fermi surfaces. In evaluating $\mathcal{P}_{I}^{a}(k)$, we will take $\bar{\mu}$ to be the arithmetic mean of $\mu_{k}$ and $\mu_{j}$, for convenience, but since the two integrals on the right-hand side of Eq. (57) go as $\log (\Lambda)$ for large momenta, choosing any other value for $\bar{\mu}$ close to the Fermi surfaces changes the value of $\mathcal{P}_{I}^{a}(k)$ only by $\mathcal{O}(\delta \mu / \Lambda)$, which we will ignore. We introduce the two null vectors, $V^{\mu}=(1, \hat{\mathbf{v}})$ and $\tilde{V}^{\mu}=(1,-\hat{\mathbf{v}})$, as is done in the High Density Effective Theory (HDET) [60]. It is also useful to define four momenta $l^{\mu} \equiv\left(p^{0}, s \hat{\mathbf{v}}\right)=p^{\mu}-(0, \bar{\mu} \hat{\mathbf{v}})$, which can be thought of as residual momenta as measured from the Fermi surface. It is easy to verify that $V \cdot l=p^{0}-s$ and $\tilde{V} \cdot l=p^{0}+s$. In the weak coupling limit, for a generic four vector $p^{\prime}$ that is small compared to $\Lambda$, the propagators in Eq. (57) simplify as follows: 


$$
\begin{aligned}
\frac{1}{\not p+\not p^{\prime}+\mu_{j}} & =\frac{\left(p^{0}+\left(p^{\prime}\right)^{0}+\mu_{j}\right) \gamma^{0}-\left(\mathbf{p}+\mathbf{p}^{\prime}\right) \cdot \vec{\gamma}}{\left(p^{0}+\left(p^{\prime}\right)^{0}+\mu_{j}-\left|\mathbf{p}+\mathbf{p}^{\prime}\right|\right)\left(p^{0}+\left(p^{\prime}\right)^{0}+\mu_{j}+\left|\mathbf{p}+\mathbf{p}^{\prime}\right|\right)} \\
& \approx \frac{\bar{\mu} \gamma^{0}-\mathbf{p} \cdot \vec{\gamma}}{\left(p^{0}+\left(p^{\prime}\right)^{0}+\bar{\mu}+\left(-\bar{\mu}+\mu_{j}\right)-|\mathbf{p}|-\mathbf{p}^{\prime} \cdot \hat{\mathbf{v}}\right)(2 \overline{\bar{\mu}})} \\
& \approx \frac{1}{2}\left(\frac{\gamma^{0}-\hat{\mathbf{v}} \cdot \vec{\gamma}}{p^{0}+\left(p^{\prime}\right)^{0}-s+\left(\mu_{j}-\bar{\mu}\right)-\mathbf{p}^{\prime} \cdot \hat{\mathbf{v}}}\right) \\
& =\frac{1}{2}\left(\frac{V \cdot \gamma}{V \cdot\left(l+p^{\prime}\right)+\left(\mu_{j}-\bar{\mu}\right)}\right)
\end{aligned}
$$

and, similarly,

$$
\begin{aligned}
\frac{1}{\not p+\not p^{\prime}-\mu_{k}} & =\frac{\left(p^{0}+\left(p^{\prime}\right)^{0}-\mu_{k}\right) \gamma^{0}-\left(\mathbf{p}+\mathbf{p}^{\prime}\right) \cdot \vec{\gamma}}{\left(p^{0}+\left(p^{\prime}\right)^{0}-\mu_{k}-\left|\mathbf{p}+\mathbf{p}^{\prime}\right|\right)\left(p^{0}+\left(p^{\prime}\right)^{0}-\mu_{k}+\left|\mathbf{p}+\mathbf{p}^{\prime}\right|\right)} \\
& \approx \frac{1}{2}\left(\frac{\gamma^{0}+\hat{\mathbf{v}} \cdot \vec{\gamma}}{p^{0}+\left(p^{\prime}\right)^{0}+s-\left(\mu_{k}-\bar{\mu}\right)+\mathbf{p}^{\prime} \cdot \hat{\mathbf{v}}}\right) \\
& =\frac{1}{2}\left(\frac{\tilde{V} \cdot \gamma}{\tilde{V} \cdot\left(l+p^{\prime}\right)-\left(\mu_{k}-\bar{\mu}\right)}\right) .
\end{aligned}
$$

Upon using these simplifications in Eq. (57), we obtain,

$$
\begin{aligned}
\mathcal{P}_{I}^{a}(k) & =\frac{\mu^{2}}{\pi^{2}}\left[\int_{-\infty}^{+\infty} \frac{d p^{0}}{2 \pi i} \int_{-\Lambda}^{\Lambda} d s \int \frac{d \hat{\mathbf{v}}}{4 \pi} \frac{1}{\left(V \cdot l-\hat{\mathbf{v}} \cdot \mathbf{q}_{I}^{a}+\delta \mu_{I}\right)\left(\tilde{V} \cdot l-\hat{\mathbf{v}} \cdot \mathbf{q}_{I}^{a}+\delta \mu_{I}\right)}\right. \\
- & \left.\int_{-\infty}^{+\infty} \frac{d p^{0}}{2 \pi i} \int_{-\Lambda}^{\Lambda} d s \int \frac{d \hat{\mathbf{v}}}{4 \pi} \frac{1}{\left(V \cdot\left(l+k_{1}\right)-\hat{\mathbf{v}} \cdot \mathbf{q}_{I}^{a}+\delta \mu_{I}\right)\left(\tilde{V} \cdot\left(l+k_{2}\right)-\hat{\mathbf{v}} \cdot \mathbf{q}_{I}^{a}+\delta \mu_{I}\right)}\right]+\left(\delta \mu_{I} \rightarrow-\delta \mu_{I}\right) .
\end{aligned}
$$

By making the changes of variables $p^{0} \rightarrow-p^{0}$, $s \rightarrow-s, \hat{\mathbf{v}} \rightarrow-\hat{\mathbf{v}}$ and $\delta \mu_{I} \rightarrow-\delta \mu_{I}$ it is easy to show that $\mathcal{P}_{I}^{a}\left(-k^{0}, \mathbf{k}\right)=\mathcal{P}_{I}^{a}\left(k^{0}, \mathbf{k}\right)$. In addition, it is clear from Eq. (56) that only the part of $\mathcal{P}_{I}^{a}(k)$ that is even under $k \rightarrow-k$ contributes to $\mathcal{S}^{\phi^{2} \Delta^{2}}$. Hence, terms in the expansion of $\mathcal{P}_{I}^{a}(k)$ proportional to odd powers of $k$ do not contribute to the effective action. Furthermore, at second order in $k$ this implies that there cannot be terms proportional to $k_{0} \mathbf{k} \phi^{2} \Delta^{2}$ in the effective action. (Terms like $k_{0}^{2} \mathbf{k}^{2} \phi^{2} \Delta^{2}$ can of course appear, but are higher order in $k$.) This is useful because we can handle the spatial and time components of $k$ independently, thereby simplifying the calculation of $\mathcal{P}_{I}^{a}(k)$.

In order to simplify the calculation we rewrite $\mathcal{P}_{I}^{a}(k)$ a little differently. Multiplying and dividing the integrand appearing in the second term in Eq. (61) (the integrand depending upon $k_{1}$ and $k_{2}$ ) by

$$
\begin{aligned}
& \left(\tilde{V} \cdot\left(l+k_{1}\right)-\hat{\mathbf{v}} \cdot \mathbf{q}_{I}^{a}+\delta \mu_{I}\right) \\
& \times\left(V \cdot\left(l+k_{2}\right)-\hat{\mathbf{v}} \cdot \mathbf{q}_{I}^{a}+\delta \mu_{I}\right)
\end{aligned}
$$

and collecting the term with numerator $(V \cdot k)(\tilde{V} \cdot k)=\left(V \cdot\left(k_{2}-k_{1}\right)\right)\left(\tilde{V} \cdot\left(k_{2}-k_{1}\right)\right)$, after some algebra we obtain

$$
\frac{2}{\left(V \cdot\left(l+k_{1}\right)-\hat{\mathbf{v}} \cdot \mathbf{q}_{I}^{a}+\delta \mu_{I}\right)\left(\tilde{V} \cdot\left(l+k_{2}\right)-\hat{\mathbf{v}} \cdot \mathbf{q}_{I}^{a}+\delta \mu_{I}\right)}=-\frac{(V \cdot k)(\tilde{V} \cdot k)}{D\left(l+k_{1}\right) D\left(l+k_{2}\right)}+\frac{1}{D\left(l+k_{1}\right)}+\frac{1}{D\left(l+k_{2}\right)},
$$


where

$$
D(l) \equiv\left(\tilde{V} \cdot l+\delta \mu_{I}-\hat{\mathbf{v}} \cdot \mathbf{q}_{I}^{a}\right)\left(V \cdot l+\delta \mu_{I}-\hat{\mathbf{v}} \cdot \mathbf{q}_{I}^{a}\right) .
$$

We can then write $\mathcal{P}_{I}^{a}(k)$ as $\mathcal{P}_{I}^{a}(k)=I_{0}+I_{1}$ with

$$
\begin{aligned}
I_{1} \equiv & \frac{1}{2} \frac{\mu^{2}}{\pi^{2}} \int \frac{d p^{0}}{2 \pi i} \int_{-\Lambda}^{\Lambda} d s \int \frac{d \hat{\mathbf{v}}}{4 \pi} \frac{(V \cdot k)(\tilde{V} \cdot k)}{D\left(l+k_{1}\right) D\left(l+k_{2}\right)}+\left(\delta \mu_{I} \rightarrow-\delta \mu_{I}\right) \\
I_{0} \equiv & \frac{\mu^{2}}{\pi^{2}} \int \frac{d p^{0}}{2 \pi i} \int_{-\Lambda}^{\Lambda} d s \int \frac{d \hat{\mathbf{v}}}{4 \pi} \frac{1}{D(l)}-\frac{1}{2} \frac{\mu^{2}}{\pi^{2}} \int \frac{d p^{0}}{2 \pi i} \int_{-\Lambda}^{\Lambda} d s \int \frac{d \hat{\mathbf{v}}}{4 \pi} \frac{1}{D\left(l+k_{1}\right)} \\
& -\frac{1}{2} \frac{\mu^{2}}{\pi^{2}} \int \frac{d p^{0}}{2 \pi i} \int_{-\Lambda}^{\Lambda} d s \int \frac{d \hat{\mathbf{v}}}{4 \pi} \frac{1}{D\left(l+k_{2}\right)}+\left(\delta \mu_{I} \rightarrow-\delta \mu_{I}\right) .
\end{aligned}
$$

The reason to separate $\mathcal{P}_{I}^{a}(k)$ as a sum of $I_{0}$ and $I_{1}$ will become clear in a moment, when we argue that $I_{0}=0$.

We now proceed to evaluate $I_{0}$ and $I_{1}$ separately. As we discussed, we can consider the spatial and the temporal parts of $k$ independently, and we begin by taking $k=(0, \mathbf{k})$ with $\mathbf{k}=\mathbf{k}_{2}-\mathbf{k}_{1}$. In this case $I_{0}$ can be expressed as a sum of three terms, each proportional (up to a prefactor $+\mu^{2} / \pi^{2}$ or $-\mu^{2} /\left(2 \pi^{2}\right)$ ) to an integral of the form

$$
\begin{aligned}
\Pi\left(\mathbf{q}_{I}^{a}, \delta \mu_{I}, p^{\prime}\right) & =\int \frac{d p^{0}}{2 \pi i} \int_{-\Lambda}^{\Lambda} d s \int \frac{d \hat{\mathbf{v}}}{4 \pi} \frac{1}{D\left(l+p^{\prime}\right)} \\
& =\int \frac{d p^{0}}{2 \pi i} \int_{-\Lambda}^{\Lambda} d s \int \frac{d \hat{\mathbf{v}}}{4 \pi} \frac{1}{\left(p^{0}-s-\hat{\mathbf{v}} \cdot \mathbf{p}^{\prime}-\hat{\mathbf{v}} \cdot \mathbf{q}_{I}^{a}+\delta \mu_{I}\right)\left(p^{0}+s+\hat{\mathbf{v}} \cdot \mathbf{p}^{\prime}-\hat{\mathbf{v}} \cdot \mathbf{q}_{I}^{a}+\delta \mu_{I}\right)}
\end{aligned}
$$

where $p^{\prime}$ is in this case a purely spatial vector, $p^{\prime}=\left(0, \mathbf{p}^{\prime}\right)$. This integral can be evaluated by following the steps outlined in Ref. [30]. We first perform a Wick rotation, $p^{0} \rightarrow i p^{4}$, and then do the $p^{4}$ integration by the method of residues, followed by the $d s$ and $d \hat{\mathbf{v}}$ integrals. For $\mathbf{p}^{\prime}=0$, the integral is calculated in Ref. [30] and is given by

$$
\Pi\left(\mathbf{q}_{I}^{a}, \delta \mu_{I}, 0\right)=\left[-1-\frac{1}{2} \log \left(\frac{\Lambda^{2}}{\left(\mathbf{q}_{I}^{a}\right)^{2}-\delta \mu_{I}^{2}}\right)+\frac{\delta \mu_{I}}{2\left|\mathbf{q}_{I}^{a}\right|} \log \left(\frac{\left|\mathbf{q}_{I}^{a}\right|+\delta \mu_{I}}{\left|\mathbf{q}_{I}^{a}\right|-\delta \mu_{I}}\right)\right] .
$$

By making the change of variables $s \rightarrow s-\hat{\mathbf{v}} \cdot \mathbf{p}^{\prime}$ in Eq. (67), we see that the integrand appearing in the definition of $\Pi\left(\mathbf{q}_{I}^{a}, \delta \mu_{I}, p^{\prime}\right)$ in Eq. (67), can be written as the integral appearing in $\Pi\left(\mathbf{q}_{I}^{a}, \delta \mu_{I}, 0\right)$, but with the limits of $s$ integration changed to $\left[-\Lambda-\hat{\mathbf{v}} \cdot \mathbf{p}^{\prime}, \Lambda-\hat{\mathbf{v}} \cdot \mathbf{p}^{\prime}\right]$. Since, $\Pi$ goes as $\log (\Lambda)$, however, this change in limits changes the value of the integrand only by a quantity of order $\mathbf{k} / \Lambda$, which we ignore. Thus,

$$
I_{0}=\frac{\mu^{2}}{\pi^{2}}\left[\Pi\left(\mathbf{q}_{I}^{a}, \delta \mu_{I}, 0\right)-\frac{1}{2} \Pi\left(\mathbf{q}_{I}^{a}, \delta \mu_{I}, 0\right)-\frac{1}{2} \Pi\left(\mathbf{q}_{I}^{a}, \delta \mu_{I}, 0\right)\right]+\left(\delta \mu_{I} \rightarrow-\delta \mu_{I}\right)=0 .
$$

The integral $I_{1}$ can be evaluated using similar steps, namely Wick rotate $p^{0} \rightarrow i p^{4}$, perform the $p^{4}$ integration by residues and then do the $d s$ and $d \hat{\mathbf{v}}$ integrals. The final result is

$$
I_{1}=\frac{\mu^{2}}{\pi^{2}}\left[\Pi\left(\mathbf{q}_{I}^{a}, \delta \mu_{I}, 0\right)-\frac{1}{2} \Pi\left(\mathbf{q}_{I}^{a}-\frac{1}{2} \mathbf{k}, \delta \mu_{I}, 0\right)-\frac{1}{2} \Pi\left(\mathbf{q}_{I}^{a}+\frac{1}{2} \mathbf{k}, \delta \mu_{I}, 0\right)\right]+\left(\delta \mu_{I} \rightarrow-\delta \mu_{I}\right) .
$$


We note that the final result depends only upon $\mathbf{k}=\mathbf{k}_{2}-\mathbf{k}_{1}$. Expanding $I_{1}$ in $\mathbf{k}$, we find

$$
\begin{aligned}
\mathcal{P}_{I}^{a}(k) & =-\mathbf{k}_{\| a}^{2} \frac{\mu^{2}}{\pi^{2}}\left[\frac{1}{4\left(\mathbf{q}_{I}^{a 2}-\delta \mu_{I}^{2}\right)}-\frac{1}{2\left(\mathbf{q}_{I}^{a}\right)^{2}}\left(1+\frac{\delta \mu_{I}}{2\left|\mathbf{q}_{I}^{a}\right|} \log \left(\frac{\left|\mathbf{q}_{I}^{a}\right|-\delta \mu_{I}}{\left|\mathbf{q}_{I}^{a}\right|+\delta \mu_{I}}\right)\right)\right] \\
& -\mathbf{k}_{\perp a}^{2} \frac{\mu^{2}}{\pi^{2}}\left[\frac{1}{4\left(\mathbf{q}_{I}^{a}\right)^{2}}\left(1+\frac{\delta \mu_{I}}{2\left|\mathbf{q}_{I}^{a}\right|} \log \left(\frac{\left|\mathbf{q}_{I}^{a}\right|-\delta \mu_{I}}{\left|\mathbf{q}_{I}^{a}\right|+\delta \mu_{I}}\right)\right)\right]+\mathcal{O}\left(k^{4}\right),
\end{aligned}
$$

where $\mathbf{k}_{\| a}$ is the component of $\mathbf{k}$ which is parallel to $\mathbf{q}_{I}^{a}, \mathbf{k}_{\| a}=\hat{\mathbf{q}}_{I}^{a}\left(\mathbf{k} \cdot \hat{\mathbf{q}}_{I}^{a}\right)$, and $\mathbf{k}_{\perp a}$ is the component perpendicular to $\mathbf{q}_{I}^{a}, \mathbf{k}_{\perp a}=\mathbf{k}-\mathbf{k}_{\| a}$. In deriving Eq. (71) we did not assume any particular relations between $\mathbf{q}_{I}^{a}$ and $\delta \mu_{I}$, but now we choose the value of $\left|\mathbf{q}_{I}^{a}\right|$ given by Eq. (15) that minimizes the free energy. Substituting Eqs. (15) and (16) into Eq. (71) simplifies $\mathcal{P}_{I}^{a}(k)$ considerably, yielding

$\mathcal{P}_{I}^{a}(k)=-\mathbf{k}_{\| a}^{2} \frac{\mu^{2}}{\pi^{2}}\left[\frac{1}{4 \delta \mu_{I}^{2}\left(\eta^{2}-1\right)}\right] \quad$ for $k=(0, \mathbf{k})$,

where we have dropped the terms of order $k^{4}$.

The final expression for $\mathcal{P}_{I}^{a}(k)$ has the following features. First, the $\mathbf{k}_{\perp a}$ term has dropped out. This means that for a single plane wave condensate, phonons that propagate in the direction orthogonal to the plane wave that forms the condensate cost no energy up to order $\left(\mathbf{k}_{\perp a}\right)^{2} \Delta^{2} \phi^{2}$. Second, we note that the coefficient in front of $\left(\mathbf{k}_{\| a}\right)^{2}$ is negative. This means that the crystal structure is stable with respect to small fluctuations in the direction of $\mathbf{q}_{I}^{a}$. (Recall that action goes like kinetic energy minus potential energy; since here $k$ is spatial, we have potential energy only meaning that decreasing the action corresponds to increasing the energy, hence stability.) This result is a direct consequence of the fact that we chose $\left|\mathbf{q}_{I}^{a}\right|$ to minimize $\alpha_{I}$, meaning that any deviation from the most favorable modulation of the condensate in the direction of $\mathbf{q}_{I}^{a}$ increases the free energy of the system by an amount of order $\Delta^{2}$.

We now evaluate $I_{0}$ and $I_{1}$ in the case where $k$ is purely temporal, namely $k=\left(k^{0}, \mathbf{0}\right)$ with $k^{0}=$ $k_{2}^{0}-k_{1}^{0}$. With these $k_{1}$ and $k_{2}$, the value of $I_{1}$ turns out to be

$$
\begin{aligned}
I_{1} & =\frac{\mu^{2}}{\pi^{2}}\left[\frac{1}{2} \log \left(\frac{\left(\delta \mu_{I}-k_{1}^{0}+\left(k^{0} / 2\right)\right)^{2}-\left(\mathbf{q}_{I}^{a}\right)^{2}}{\left(\delta \mu_{I}-k_{1}^{0}\right)^{2}-\left(\mathbf{q}_{I}^{a}\right)^{2}}\right)+\left(\delta \mu_{I}-k_{1}^{0}+\left(k^{0} / 2\right)\right) \log \left(\frac{\delta \mu_{I}-k_{1}^{0}+\left(k^{0} / 2\right)+\left|\mathbf{q}_{I}^{a}\right|}{\delta \mu_{I}-k_{1}^{0}+\left(k^{0} / 2\right)-\left|\mathbf{q}_{I}^{a}\right|}\right)\right. \\
& -\left(\delta \mu_{I}-k_{1}^{0}\right) \log \left(\frac{\delta \mu_{I}-k_{1}^{0}+\left|\mathbf{q}_{I}^{a}\right|}{\delta \mu_{I}-k_{1}^{0}-\left|\mathbf{q}_{I}^{a}\right|}\right)+\frac{1}{2} \log \left(\frac{\left(\delta \mu_{I}-k_{2}^{0}-\left(k^{0} / 2\right)\right)^{2}-\left(\mathbf{q}_{I}^{a}\right)^{2}}{\left(\delta \mu_{I}-k_{2}^{0}\right)^{2}-\left(\mathbf{q}_{I}^{a}\right)^{2}}\right) \\
& \left.+\left(\delta \mu_{I}-k_{2}^{0}-\left(k^{0} / 2\right)\right) \log \left(\frac{\delta \mu_{I}-k_{2}^{0}-\left(k^{0} / 2\right)+\left|\mathbf{q}_{I}^{a}\right|}{\delta \mu_{I}-k_{2}^{0}-\left(k^{0} / 2\right)-\left|\mathbf{q}_{I}^{a}\right|}\right)-\left(\delta \mu_{I}-k_{2}^{0}\right) \log \left(\frac{\delta \mu_{I}-k_{2}^{0}+\left|\mathbf{q}_{I}^{a}\right|}{\delta \mu_{I}-k_{2}^{0}-\left|\mathbf{q}_{I}^{a}\right|}\right)\right]+\left(\delta \mu_{I} \rightarrow-\delta \mu_{I}\right) .
\end{aligned}
$$

Although, it appears that Eq. (73) does not depend solely on $k_{2}^{0}-k_{1}^{0}$, upon expanding in small $k_{1}^{0}$ and $k_{2}^{0}$, we find,

$$
I_{1}=\left(k^{0}\right)^{2} \frac{\mu^{2}}{\pi^{2}} \frac{1}{4\left(\left(\mathbf{q}_{I}^{a}\right)^{2}-\delta \mu_{I}^{2}\right)}+\mathcal{O}\left(k^{4}\right) \approx\left(k^{0}\right)^{2} \frac{\mu^{2}}{\pi^{2}} \frac{1}{4 \delta \mu_{I}^{2}\left(\eta^{2}-1\right)} .
$$

Turning now to $I_{0}$, this quantity is given by

$$
I_{0}=\frac{\mu^{2}}{\pi^{2}}\left[\Pi\left(\mathbf{q}_{I}^{a}, \delta \mu_{I}, 0\right)-\frac{1}{2} \Pi\left(\mathbf{q}_{I}^{a}, \delta \mu_{I}, k_{1}\right)-\frac{1}{2} \Pi\left(\mathbf{q}_{I}^{a}, \delta \mu_{I}, k_{2}\right)\right]
$$


where $k_{i}=\left(k_{i}^{0}, \mathbf{0}\right)$. When its third argument is a purely temporal four-vector, $\Pi$ is given by

$$
\Pi\left(\mathbf{q}_{I}^{a}, \delta \mu_{I}, p^{\prime}\right)=\int \frac{d p^{0}}{2 \pi i} \int_{-\Lambda}^{\Lambda} d s \int \frac{d \hat{\mathbf{v}}}{4 \pi} \frac{1}{\left(p^{0}+p^{\prime 0}-s-\hat{\mathbf{v}} \cdot \mathbf{q}_{I}^{a}+\delta \mu_{I}\right)\left(p^{0}+p^{\prime 0}+s-\hat{\mathbf{v}} \cdot \mathbf{q}_{I}^{a}+\delta \mu_{I}\right)},
$$

where $p^{\prime}=\left(p^{\prime 0}, \mathbf{0}\right)$. It is apparent from Eq. (766) that by making the change of variables $p^{0} \rightarrow p^{0}+p^{\prime 0}$ we obtain $\Pi\left(\mathbf{q}_{I}^{a}, \delta \mu_{I}, p^{\prime 0}\right)=\Pi\left(\mathbf{q}_{I}^{a}, \delta \mu_{I}, 0\right)$, leading us to conclude from Eq. (75) that $I_{0}=0$. We advise the reader that in order to obtain the result $I_{0}=0$ by the approach that we have employed in a straightforward manner, it is important to shift $p_{0}$ before Wick rotating. (Note that if we calculate $I_{0}$ using dimensional regularization we find $I_{0}=0$ in agreement with what we obtained by change of variables.)
Finally, therefore, with $I_{0}=0$ we obtain

$$
\mathcal{P}_{I}^{a}(k)=\left(k^{0}\right)^{2} \frac{\mu^{2}}{\pi^{2}} \frac{1}{4 \delta \mu_{I}^{2}\left(\eta^{2}-1\right)} \quad \text { for } k=\left(k^{0}, \mathbf{0}\right)
$$

We see that this comes with a positive sign, as is appropriate for a kinetic energy term.

Substituting the expressions given in Eqs. (72) and (77) back into the action (56), we obtain

$$
\mathcal{S}^{\phi^{2} \Delta^{2}}=\sum_{I} \sum_{\mathbf{q}_{I}^{a}} \int \frac{d^{4} k}{(2 \pi)^{4}} \phi_{I}^{a}(k) \phi_{I}^{a}(k)\left[k_{0}^{2}-\left(\mathbf{k}_{\| a}\right)^{2}\right] \frac{\mu^{2}}{\pi^{2}} \frac{\left|\Delta_{I}\right|^{2}}{4 \delta \mu_{I}^{2}\left(\eta^{2}-1\right)},
$$

where $\mathbf{k}_{\| a}=\hat{\mathbf{q}}_{I}^{a}\left(\mathbf{k} \cdot \hat{\mathbf{q}}_{I}^{a}\right)$ and $\phi_{I}^{a}(k)=\mathbf{u}_{I} \cdot\left(2 \mathbf{q}_{I}^{a}\right)$. Inverse Fourier transforming back to position space, and taking out a factor of half for future convenience, we obtain the effective action for the displacement fields:

$$
\mathcal{S}[\mathbf{u}]=\frac{1}{2} \int d^{4} x \sum_{I} \frac{\mu^{2}}{\pi^{2}} \frac{2\left|\Delta_{I}\right|^{2} \eta^{2}}{\left(\eta^{2}-1\right)} \sum_{\mathbf{q}_{I}^{a}}\left[\partial_{0}\left(\hat{\mathbf{q}}_{I}^{a} \cdot \mathbf{u}_{I}\right) \partial_{0}\left(\hat{\mathbf{q}}_{I}^{a} \cdot \mathbf{u}_{I}\right)-\left(\hat{\mathbf{q}}_{I}^{a} \cdot \vec{\partial}\right)\left(\hat{\mathbf{q}}_{I}^{a} \cdot \mathbf{u}_{I}\right)\left(\hat{\mathbf{q}}_{I}^{a} \cdot \vec{\partial}\right)\left(\hat{\mathbf{q}}_{I}^{a} \cdot \mathbf{u}_{I}\right)\right] .
$$

This is the low energy effective action for phonons in any crystalline color superconducting phase, valid to second order in derivatives, to second order in the gap parameters $\Delta_{I}$ 's and to second order in the phonon fields $u_{I}$. This is the central technical result of our paper.

Because we are interested in long wavelength, small amplitude, phonon excitations, expanding to second order in derivatives and to second order in the $u_{I}$ is satisfactory in every respect. Not so for the expansion to second order in the $\Delta_{I}$. As we have discussed previously, the Ginzburg-Landau approximation is at the point of breaking down in the most favorable CubeX and 2Cube $45 \mathrm{z}$ crystal structures. Before proceeding, we therefore ask what kind of corrections to (78) will arise at higher order in $\Delta$. The first thing to note is that in the weak coupling limit $\mu$ appears only as an overall factor of $\mu^{2}$ in front of the fermion loop integrals. After simplify- ing the fermionic propagators as in (59) and (60) and taking $\Lambda$ to $\infty$, the only two independent dimensionful quantities that remain in the integrals are $k$ and $\delta \mu_{I}$. (Recall that $\left|\mathbf{q}_{I}\right|$ is given by $\eta \delta \mu_{I}$ and so is not independent.) Since we found the action only up to terms which are second order in the derivatives and second order in $\Delta$, to ensure the Lagrangian density has dimension four, only a dimensionless factor can multiply $\mu^{2}\left|\Delta_{I}\right|^{2} \partial^{2} \mathbf{u}_{I}^{2}$, as we can see is true in Eq. (79). Higher powers of $\Delta^{2}$ will appear in Eq. (78) in combination with higher compensating powers of $\delta \mu^{-2}$. Consequently, there will be corrections to the coefficients of $k_{0}^{2}$ and $\left(\mathbf{k}_{\| a}\right)^{2}$ in (78) suppressed by factors of $\left(\Delta^{2} / \delta \mu^{2}\right)$ relative to the leading order result that we have obtained. In addition, there will be new terms. There is no reason to expect that the coefficient of $\left(\mathbf{k}_{\perp a}\right)^{2}$ will remain zero at $\mathcal{O}\left(\mu^{2}\left|\Delta_{I}\right|^{4}\left(\partial \mathbf{u}_{I}\right)^{2} / \delta \mu^{2}\right)$. Finally, we 
see that there are no terms in (78) that "mix" the different $\mathbf{u}_{I}(k)$. This follows from the color-flavor structure of the condensate as discussed above. At higher order, there will be terms proportional to $\mu^{2}\left|\Delta_{I} \Delta_{J}\right|^{2} \partial \mathbf{u}_{I} \partial \mathbf{u}_{J} / \delta \mu^{2}$, which do "mix" the different $\mathbf{u}_{I}$ 's.

With the phonon effective action now in hand, in Section IV we shall relate the coefficients of the terms in $\mathcal{S}(\mathbf{u})$ involving spatial derivatives acting on the displacement fields to the shear modulus of crystalline color superconducting quark matter with specified crystal structures.

\section{EXTRACTING THE SHEAR MODULUS}

We see from Eq. (79) that the action of the phonon fields, $\mathcal{S}(\mathbf{u})$, is a sum of two terms: the kinetic energy, which has time derivatives acting on the fields $\mathbf{u}$, and the potential energy, which has spatial derivatives acting on $\mathbf{u}$. From the basic theory of elastic media 61], the potential energy is related to the elastic moduli that describe the energy cost of small deformations of the crystal. In this Section, we present this relation explicitly and calculate the shear modulus for the CubeX and 2Cube $45 \mathrm{z}$ crystal structures.

\section{A. Generalities}

Let there be a set of displacement fields $\mathbf{u}_{I}$ propagating in a crystalline color superconducting material. (We will set the problem up in the general case where all the $\Delta_{I}$ are nonzero, meaning that $I$ runs from 1 to 3.) The kinetic energy density for the displacement fields takes the form

$$
\mathcal{K}=\frac{1}{2} \sum_{I J} \sum_{m n} \rho_{I J}^{m n}\left(\partial_{0} \mathbf{u}_{I}^{m}\right)\left(\partial_{0} \mathbf{u}_{J}^{n}\right),
$$

where $\mathbf{u}_{I}^{m}, \mathbf{u}_{J}^{n}$ are the space components of the vectors $\mathbf{u}_{I}$ and $\mathbf{u}_{J}$ respectively. (We will use the indices $m, n, u$ and $v$ to represent spatial indices in the following). As we are working only to order $\Delta^{2}$, the only nonzero components of $\rho_{I J}^{m n}$ are those with $I=J$. We will choose the direction of the axes, $x, y$ and $z$ such that for every $I$ and $J, \rho_{I J}^{m n}$ is diagonal in the $m$ and $n$ indices and we will denote the diagonal components of $\rho_{I J}^{m n}$ by $\rho_{I}^{m}$. We can then rewrite the kinetic energy density as

$$
\mathcal{K}=\frac{1}{2} \sum_{I} \sum_{m} \rho_{I}^{m}\left(\partial_{0} \mathbf{u}_{I}^{m}\right)\left(\partial_{0} \mathbf{u}_{I}^{m}\right) .
$$

At higher orders in $\Delta^{2}$, we could need to choose a new linear combination of fields $\tilde{\mathbf{u}}_{I}^{m}=A_{I J}^{m n} \mathbf{u}_{J}^{n}$ to render the kinetic energy diagonal in the $I J$ and $m n$ indices.

The potential energy density to quadratic order in the displacement fields can be written as

$$
\mathcal{U}=\frac{1}{2} \sum_{I J} \sum_{\substack{m n \\ u v}} \lambda_{I J}^{m u n v} \frac{\partial \mathbf{u}_{I}^{m}}{\partial x^{u}} \frac{\partial \mathbf{u}_{J}^{n}}{\partial x^{v}},
$$

where $\lambda_{I J}^{\text {munv }}$ is the elastic modulus tensor. The components of the tensor $\partial \mathbf{u}_{I}^{m} / \partial x^{n}$ that are antisymmetric in the $m n$ space indices are related to rigid rotations. The symmetric components of the tensor, namely the "strain tensor"

$$
s_{I}^{m u}=\frac{1}{2}\left(\frac{\partial \mathbf{u}_{I}^{m}}{\partial x^{u}}+\frac{\partial \mathbf{u}_{I}^{u}}{\partial x^{m}}\right),
$$

tell us about deformations of the medium. In the previous Section we have shown that, to order $\Delta^{2}$, there is no interaction between the displacement fields $\mathbf{u}_{I}$ and $\mathbf{u}_{J}$ with $I$ different from $J$. Therefore $\lambda_{I J}^{\text {munv }}$ is diagonal in the $I$ and $J$ indices and, denoting the diagonal entries by $\lambda_{I}^{\text {munv }}$, we find

$$
\mathcal{U}=\frac{1}{2} \sum_{I} \sum_{\substack{m n \\ u v}} \lambda_{I}^{m u n v} s_{I}^{m u} s_{I}^{n v} .
$$

Next, we define the stress tensor acting on the crystal $I$ as 61]

$$
\sigma_{I}^{m u}=\frac{\partial \mathcal{U}}{\partial s_{I}^{m u}},
$$

which is symmetric in its spatial indices. For a potential $\mathcal{U}$ that is quadratic in the displacement fields and is given by (84), the stress tensor is

$$
\sigma_{I}^{m u}=\lambda_{I}^{m u n v} s_{I}^{n v} .
$$

The diagonal components of $\sigma$ are proportional to the compression exerted on the system and are therefore related to the bulk modulus of the crystalline color superconducting quark matter. Since unpaired quark matter has a pressure $\sim \mu^{4}$, it gives a contribution to the bulk modulus that completely 
overwhelms the contribution from the condensation into a crystalline phase, which is of order $\mu^{2} \Delta^{2}$. We shall therefore not calculate the bulk modulus. On the other hand, the response to shear stress arises only because of the presence of the crystalline condensate.

The shear modulus can be defined as follows. Imagine exerting a static external stress $\sigma_{I}$ having only an off-diagonal component, meaning $\sigma_{I}^{m u} \neq 0$ for a pair of space directions $m \neq u$, and all the other components of $\sigma$ are zero. The system will respond with a strain $s_{I}^{n v}$ satisfying (85). The shear modulus in the $m u$ plane, $\nu_{I}^{m u}$, is defined as half the ratio of the stress to the strain:

$$
\nu_{I}^{m u}=\frac{\sigma_{I}^{m u}}{2 s_{I}^{m u}},
$$

where the indices $m$ and $u$ are not summed. For a quadratic potential, with $\sigma_{I}^{m n}$ given by (86), the shear modulus is

$$
\nu_{I}^{m u}=\frac{\lambda_{I}^{m u n v} s_{I}^{n v}}{2 s_{I}^{m u}}
$$

where $n$ and $v$ are summed but $m$ and $u$ are not. For all the crystal structures that we shall consider below, the only nonzero entries in $\lambda^{\text {munv }}$ with $m \neq u$ are the $\lambda^{\text {mumu }}$ entries, meaning that (86) simplifies even further to

$$
\nu_{I}^{m u}=\frac{1}{2} \lambda_{I}^{m u m u}
$$

again with $m$ and $u$ not summed.

Putting Eq. (81) and Eq. (84) together, the action for the displacement fields can be written as

$$
\mathcal{S}[\mathbf{u}]=\int d^{4} x(\mathcal{K}-\mathcal{U})=\frac{1}{2} \int d^{4} x\left(\sum_{I} \sum_{m} \rho_{I}^{m}\left(\partial_{0} \mathbf{u}_{I}^{m}\right)\left(\partial_{0} \mathbf{u}_{I}^{m}\right)-\sum_{I} \sum_{\substack{m n \\ u v}} \lambda_{I}^{m u n v} s_{I}^{m u} s_{I}^{n v}\right) .
$$

The equations of motion obtained by extremizing the action $\mathcal{S}[\mathbf{u}]$ with respect to the displacement fields $\mathbf{u}$ are

$$
\rho_{I}^{m} \frac{\partial^{2} \mathbf{u}_{I}^{m}}{\partial t^{2}}=\lambda_{I}^{m u n v} \partial_{u} \partial_{n} u_{I}^{v},
$$

where $I$ and $m$ are not summed. The dispersion relations are found by solving

$$
\operatorname{Det}\left[\rho_{I}^{m} k_{0}^{2} \delta_{m n}-\lambda_{I}^{m u n v} k_{u} k_{v}\right]=0
$$

for all $I$, where $m$ is again not summed.

\section{B. Elastic moduli of crystalline phases}

In order to set up the extraction of the elastic moduli of crystalline phases, we need to rewrite the action (79) for a generic crystalline phase in a form which makes comparison to (90) straightforward. Writing the spatial indices in Eq. (79) explicitly, we obtain the low energy phonon effective action in the form

$$
S[\mathbf{u}]=\frac{1}{2} \int d^{4} x \sum_{I} \kappa_{I}\left[\left(\sum_{\mathbf{q}_{I}^{a}}\left(\hat{q}_{I}^{a}\right)^{m}\left(\hat{q}_{I}^{a}\right)^{n}\right)\left(\partial_{0} \mathbf{u}_{I}^{m}\right)\left(\partial_{0} \mathbf{u}_{I}^{n}\right)-\left(\sum_{\mathbf{q}_{I}^{a}}\left(\hat{q}_{I}^{a}\right)^{m}\left(\hat{q}_{I}^{a}\right)^{u}\left(\hat{q}_{I}^{a}\right)^{n}\left(\hat{q}_{I}^{a}\right)^{v}\right)\left(\partial_{u} \mathbf{u}_{I}^{m}\right)\left(\partial_{v} \mathbf{u}_{I}^{n}\right)\right]
$$

where we have defined

$$
\kappa_{I} \equiv \frac{2 \mu^{2}\left|\Delta_{I}\right|^{2} \eta^{2}}{\pi^{2}\left(\eta^{2}-1\right)} .
$$

For a given crystal structure, upon evaluating the sums in (93) and then using the definition (83) to 
compare (93) to (90), we can extract expressions for the $\lambda$ tensor and thence for the shear moduli. The quantity $\kappa$ is related to the elastic modulus for a condensate whose "crystal" structure is just a single plane wave, as discussed in Appendix B

In the next two Subsections, we will calculate the shear modulus for the CubeX and 2Cube45z crystals. We will not discuss the expression for the kinetic energy density, $\mathcal{K}$, but it is easy (and necessary) to check that in each case below we have chosen our axes such that $\mathcal{K}$ only contains terms that are diagonal in the spatial indices $m$ and $n$.

Note that henceforth we set $\Delta_{1}=0$ and $\Delta_{2}=$ $\Delta_{3}=\Delta$, meaning that

$$
\kappa_{2}=\kappa_{3} \equiv \kappa=\frac{2 \mu^{2}|\Delta|^{2} \eta^{2}}{\pi^{2}\left(\eta^{2}-1\right)} \simeq 0.664 \mu^{2}\left|\Delta^{2}\right| .
$$

The shear moduli that we evaluate each take the form of a dimensionless constant times $\kappa$.

\section{Shear modulus for the CubeX crystal}

Orienting the axes as shown in the left panel of Fig. 1, we have $\left\{\hat{\mathbf{q}}_{2}\right\}=\{(1 / \sqrt{3})( \pm \sqrt{2}, 0, \pm 1)\}$ and $\left\{\hat{\mathbf{q}}_{3}\right\}=\{(1 / \sqrt{3})(0, \pm \sqrt{2}, \pm 1)\}$. Calculating the relevant sums and substituting in (93), we find that the potential energy is given by

$$
\begin{aligned}
\mathcal{U}= & \frac{4}{9} \kappa\left(4\left(s_{2}^{x x}\right)^{2}+\left(s_{2}^{z z}\right)^{2}\right)+\frac{16}{9} \kappa\left(s_{2}^{x x} s_{2}^{z z}\right)+\frac{16}{9} \kappa\left(\left(s_{2}^{x z}\right)^{2}+\left(s_{2}^{z x}\right)^{2}\right) \\
& +\frac{4}{9} \kappa\left(4\left(s_{3}^{y y}\right)^{2}+\left(s_{3}^{z z}\right)^{2}\right)+\frac{16}{9} \kappa\left(s_{3}^{y y} s_{3}^{z z}\right)+\frac{16}{9} \kappa\left(\left(s_{3}^{y z}\right)^{2}+\left(s_{3}^{z y}\right)^{2}\right) .
\end{aligned}
$$

Recall that the only components of the stress tensor that are relevant to the calculation of the shear modulus are given by $\partial \mathcal{U} / \partial s_{I}^{m u}$ for $m \neq u$. These are

$$
\sigma_{2}^{x z}=\sigma_{2}^{z x}=\frac{32}{9} \kappa s_{2}^{z x}
$$

and

$$
\sigma_{3}^{y z}=\sigma_{3}^{z y}=\frac{32}{9} \kappa s_{3}^{z y}
$$

from which we obtain

$$
\nu_{2}^{x z}=\nu_{2}^{z x}=\frac{\sigma_{2}^{z x}}{2 s_{2}^{z x}}=\frac{16}{9} \kappa
$$

and

$$
\nu_{3}^{y z}=\nu_{3}^{z y}=\frac{\sigma_{3}^{z y}}{2 s_{3}^{z y}}=\frac{16}{9} \kappa .
$$

We can display the result succinctly by writing two shear matrices $\nu_{2}$ and $\nu_{3}$, which have only offdiagonal entries and are symmetric in the spatial indices:

$$
\nu_{2}=\frac{16}{9} \kappa\left(\begin{array}{ccc}
0 & 0 & 1 \\
0 & 0 & 0 \\
1 & 0 & 0
\end{array}\right), \quad \nu_{3}=\frac{16}{9} \kappa\left(\begin{array}{lll}
0 & 0 & 0 \\
0 & 0 & 1 \\
0 & 1 & 0
\end{array}\right) \text {. }
$$

The zeroes in these matrices are easily understood. The $\Delta_{2}$ crystal is translation invariant in the $y$ direction, because all the wave vectors in the set $\left\{\mathbf{q}_{2}\right\}$ lie in the $x z$-plane. This means that the $x y$ and $y z$-components of $\nu_{2}$ are zero. The only nonzero shear modulus is that for shear in the $x z$-plane. Note also that the $\Delta_{2}$ crystal has nonzero $\lambda_{3}^{x x x x}$ and $\lambda_{3}^{z z z z}$, meaning that it has a nonzero Young's modulus for compression or stretching in the $x$ - and $z$-directions confirming that, as the shear modulus indicates, it is rigid against deformations in the $x z$-plane. Similarly, the $\Delta_{3}$ crystal is translation invariant in the $x$-direction, meaning that the only nonzero component of the shear modulus $\nu_{3}$ is that for shear in the yz-plane.

The vortices in rotating crystalline color superconducting quark matter have currents of $u, d$ and $s$ quark-number flowing around them, meaning that the phase of both the $\Delta_{2}$ and $\Delta_{3}$ condensates winds 
once by $2 \pi$ around a rotational vortex, and meaning that both $\Delta_{2}$ and $\Delta_{3}$ vanish at the core of the vortex. This in turn means that it will be free energetically favorable for the vortices to be pinned at places where the $\Delta_{2}$ and $\Delta_{3}$ condensates already vanish in the absence of a vortex. The $\Delta_{2}$ crystal has two families of nodal planes where $\Delta_{2}(\mathbf{r})$ vanishes. One class of nodal planes are parallel to the $x y$-plane and are located at $z=((2 n+1) \pi \sqrt{3}) /(4 q)$, where $n$ is an integer. The others are parallel to the $y z$-plane and are located at $x=((2 n+1) \pi \sqrt{6}) /(4 q)$. Similarly, the $\Delta_{3}$ crystal has nodes along $z=((2 n+1) \pi \sqrt{3}) /(4 q)$ and $y=((2 n+1) \pi \sqrt{6}) /(4 q)$. So, we expect that the most favorable location of the vortices will be within the common nodal planes of the $\Delta_{2}$ and $\Delta_{3}$ condensates, namely, $z=((2 n+1) \pi \sqrt{3}) /(4 q)$. If these vortices are oriented in the $x$-direction, they will preferentially be located (i.e. will be pinned at) at $x=((2 n+1) \pi \sqrt{6}) /(4 q)$. And, if the vortices in an array of vortices oriented in the $x$-direction try to move apart (i.e. move in the $y z$-plane) as the rotation slows, in order to move they will have to shear the $\Delta_{2}$ crystal which has a nonzero $\nu_{3}^{y z}$. Similarly, if the vortices are oriented in the $y$-direction, they will preferentially be located (i.e. will be pinned at) at $y=((2 n+1) \pi \sqrt{6}) /(4 q)$. And, if the vortices in an array of vortices oriented in the $y$-direction try to move apart (i.e. move in the $x z$-plane), they will have to shear the $\Delta_{3}$ crystal which has a nonzero $\nu_{3}^{x z}$. Thus, the nonzero shear moduli that we have found in (101) are sufficient to ensure that vortices pinned within the CubeX phase are pinned to a rigid structure, with the relevant shear modulus having a magnitude $16 \kappa / 9$.

We note as an aside that further evidence for the rigidity of the CubeX crystal can be found by evaluating the phonon velocities and showing that at long wavelengths the velocity of transverse phonons (which are found in a rigid solid but not in a fluid) is comparable to that of the longitudinal phonons which are found in both fluids and solids. We will evaluate the velocities of the longitudinal phonons upon ignoring the existence of longitudinal oscillations in the gapless fermions, which have velocity $1 / \sqrt{3}$ in the limit of weak coupling. For this reason, the longitudinal phonon velocity that we calculate should be seen only as a benchmark against which to compare the transverse phonon velocity. The true sound modes would be linear combinations of the longitudinal phonons and the fermionic sound waves, which must in reality be coupled. This complication does not arise for transverse phonons: the fluid of gapless fermions has no transverse sound waves; they can only arise as excitations of a rigid structure, like the crystalline condensate we analyze. Consider as an example the phonons of the $\Delta_{2}$-crystal. From the dispersion relations (92) it is easy to show using $\rho_{2}^{x}=8 \kappa / 3, \rho_{2}^{y}=0$ and $\rho_{2}^{z}=4 \kappa / 3$ that longitudinal phonons propagating in the $x$-direction have $v=\sqrt{2 / 3}$ while transverse phonons propagating in this direction have the same $v=\sqrt{2 / 3}$. For propagation in the $z$-direction, both modes turn out to have $v=\sqrt{1 / 3}$. For propagation in other directions, there are two phonon modes with differing velocities.

\section{Shear modulus for the 2 Cube $45 \mathrm{z}$ crystal}

Let us orient the coordinate axes such that $\left\{\hat{\mathbf{q}}_{2}\right\}$ contains the eight wave vectors $(1 / \sqrt{3})( \pm 1, \pm 1, \pm 1)$ and $\left\{\hat{\mathbf{q}}_{3}\right\}$ contains $(1 / \sqrt{3})( \pm \sqrt{2}, 0, \pm 1) \cup(1 / \sqrt{3})(0, \pm \sqrt{2}, \pm 1)$. These wave vectors are shown in the right panel of Fig. 1 . The potential energy is given by

$$
\begin{aligned}
\mathcal{U}= & \frac{8}{9} \kappa\left(\left(s_{2}^{x x}\right)^{2}+\left(s_{2}^{y y}\right)^{2}+\left(s_{2}^{z z}\right)^{2}\right)+\frac{16}{9} \kappa\left(\left(s_{2}^{x x}\right)\left(s_{2}^{z z}\right)+\left(s_{2}^{y y}\right)\left(s_{2}^{z z}\right)+\left(s_{2}^{x x}\right)\left(s_{2}^{y y}\right)\right) \\
& +\frac{16}{9} \kappa\left(\left(s_{2}^{x y}\right)^{2}+\left(s_{2}^{y x}\right)^{2}+\left(s_{2}^{y z}\right)^{2}+\left(s_{2}^{z y}\right)^{2}+\left(s_{2}^{x z}\right)^{2}+\left(s_{2}^{z x}\right)^{2}\right) \\
& +\frac{8}{9} \kappa\left(2\left(s_{3}^{x x}\right)^{2}+2\left(s_{3}^{y y}\right)^{2}+\left(s_{3}^{z z}\right)^{2}\right)+\frac{16}{9} \kappa\left(\left(s_{3}^{y y}\right)\left(s_{3}^{z z}\right)+\left(s_{3}^{x x}\right)\left(s_{3}^{z z}\right)\right) \\
& +\frac{16}{9} \kappa\left(\left(s_{3}^{x z}\right)^{2}+\left(s_{3}^{z x}\right)^{2}+\left(s_{3}^{y z}\right)^{2}+\left(s_{3}^{z y}\right)^{2}\right)
\end{aligned}
$$


from which one can read off the nonzero entries of the $\lambda_{I}^{\text {munv }}$ tensors for $I=2$ and $I=3$. In the case of $\Delta_{2}$, where the axes are oriented perpendicular to the nodal planes of the crystal, the form of $\lambda_{2}^{\text {munv }}$ for the cubic crystal are easily inferred from the symmetries of the cube 61]. There are in general only three independent nonzero entries in $\lambda_{2}^{\text {munv }}$, corresponding to the terms read from (102) with the form $\lambda_{2}^{m m m m}$, $\lambda_{2}^{m m n n}$ and $\lambda_{2}^{m u m u}$. The form of $\lambda_{2}^{m u n v}$ read from (102) is therefore valid to all orders in $\Delta_{2}$, although of course the values of the coefficients, including in particular the equality between the $\lambda_{2}^{m m n n}$ and $\lambda_{2}^{m u m u}$ coefficients, will receive corrections at higher order. Finally, note that $\lambda_{3}^{\text {munv }}$ read from (102) is obtained from $\lambda_{2}^{\text {munv }}$ by rotating this tensor by $45^{\circ}$ about the $z$-axis. Note that $\lambda_{3}^{x y x y}=\lambda_{3}^{y x y x}$ vanishes. This is a consequence (after the $45^{\circ}$ rotation) of the equality of $\lambda_{2}^{m m n n}$ and $\lambda_{2}^{m u m u}$ in the $\Delta_{2}$ crystal, and is therefore not expected to persist at higher order in $\Delta_{3}$.

As in the previous subsection, we extract the $\sigma_{I}$ tensors and the matrices of shear moduli $\nu_{I}$ from the potential $\mathcal{U}$ of (102), obtaining in this case

$$
\nu_{2}=\frac{16}{9} \kappa\left(\begin{array}{lll}
0 & 1 & 1 \\
1 & 0 & 1 \\
1 & 1 & 0
\end{array}\right), \quad \nu_{3}=\frac{16}{9} \kappa\left(\begin{array}{lll}
0 & 0 & 1 \\
0 & 0 & 1 \\
1 & 1 & 0
\end{array}\right) \text {. }
$$

As discussed above, there is no symmetry reason for $\nu_{3}^{x y}=0$, so we expect that this component of the shear modulus is nonzero at order $\Delta^{4} / \delta \mu^{2}$. Note that if we were to rotate our coordinate axes by $45^{\circ}$ about the $z$-axis, it would be $\nu_{3}$ that has the zero entry while $\nu_{2}$ would have all off-diagonal entries nonzero. (To confirm this, rotate the $\lambda_{I}^{\text {munv }}$ tensors and re-extract the $\nu_{I}$ matrices, which are not tensors.)

The $\Delta$ crystal has nodes along $x=((2 n+$ 1) $\pi \sqrt{3}) /(4 q), y=((2 n+1) \pi \sqrt{3}) /(4 q)$ and $z=$ $((2 n+1) \pi \sqrt{3}) /(4 q)$. The $\Delta_{3}$ crystal has nodes along $x \pm y=((2 n+1) \pi \sqrt{6}) /(4 q)$ and $z=((2 n+$ 1) $\pi \sqrt{3}) /(4 q)$. The nodes common to both lie along the $z=((2 n+1) \pi \sqrt{3}) /(4 q)$ planes. We therefore expect that the crystal will orient itself relative to the rotation axis such that rotation vortices lie within these planes. Depending on their orientation within the planes, they could be pinned where the perpendicular nodal planes of either the $\Delta_{2}$ or the $\Delta_{3}$ crystals intersect the $z=((2 n+1) \pi \sqrt{3}) /(4 q)$ planes.

We learn from our analysis that the crystals are weaker (smaller shear modulus) with respect to shear in certain planes. We saw this explicitly for $\nu_{3}^{x y}$, which is zero to order $\Delta_{3}^{2}$ and thus presumably weaker although nonzero when higher order terms are included. The same will apply to shear in any plane obtained from this one by a symmetry transformation of the crystal, and will apply to the analogous planes for the $\Delta_{2}$ crystal. Note, however, that in the 2Cube $45 \mathrm{z}$ structure the weak planes for the $\Delta_{2}$ and $\Delta_{3}$ crystals do not coincide. This means that if it so happens that motion of a rotational vortex in a certain direction is only impeded by the weaker shear modulus of the $\Delta_{2}$ crystal, it will in fact be obstructed by the stronger shear modulus of the $\Delta_{3}$ crystal, or vice versa. Thus, the relevant shear modulus in the analysis of vortex pinning and pulsar glitches is the stronger one, which we find to be $16 \kappa / 9$ to order $\Delta^{2}$.

As in Section IV.C, we can find further evidence for the rigidity of the 2 Cube $45 z$ crystal by evaluating the velocity of the transverse and longitudinal phonons. Considering the $\Delta_{2}$-crystal as an example, from the dispersion relations (92) and $\rho_{2}^{x}=\rho_{2}^{y}=$ $\rho_{2}^{z}=8 \kappa / 3$ we find that for propagation in the $x$ - or $y$ - or $z$-direction the longitudinal phonon mode and the two transverse phonon modes all have $v=\sqrt{1 / 3}$. For propagation in the $x \pm z$ directions, the longitudinal mode has $v=\sqrt{2 / 3}$ while one transverse mode has $v=\sqrt{1 / 3}$ and the other transverse mode, corresponding to transverse oscillations for which the restoring force would be given by the component of the shear modulus which vanishes at order $\Delta^{2}$, turns out indeed to have $v=0$. We see that the velocity of both the longitudinal and transverse phonons is anisotropic, as expected in a crystal, and see that they are comparable in magnitude, confirming that the nonzero components of the shear moduli are as large as the longitudinal elastic moduli, as expected for a very rigid body.

\section{CONCLUSION}

\section{A. The rigidity of crystalline color superconducting quark matter}

We have calculated the shear moduli of crystalline color superconducting quark matter with the CubeX and 2Cube $45 z$ crystal structures. Within the Ginzburg-Landau analysis of Ref. [20], one or other of these crystal structures is favored over unpaired quark matter and over spatially uniform 
paired phases like the CFL phase in the wide regime of densities given in Eq. (11). As we have explained in Sections IV.C and IV.D, in both these structures the components of the shear moduli that make the crystals rigid with respect to vortices pinned within them take on the same value to order $\Delta^{2}$, given by

$$
\nu_{\mathrm{CQM}}=\frac{16}{9} \kappa
$$

with $\kappa$ defined by (95). Evaluating $\kappa$ yields

$$
\nu_{\mathrm{CQM}}=2.47 \mathrm{MeV} / \mathrm{fm}^{3}\left(\frac{\Delta}{10 \mathrm{MeV}}\right)^{2}\left(\frac{\mu}{400 \mathrm{MeV}}\right)^{2}
$$

for the shear moduli of crystalline quark matter with these two crystal structures. If quark matter is found within neutron stars, it is reasonable to estimate that its quark number chemical potential will lie in the range

$$
350 \mathrm{MeV}<\mu<500 \mathrm{MeV} .
$$

The gap parameter $\Delta$ is less well known. According to the Ginzburg-Landau calculations of Ref. [20], $\Delta / \Delta_{0}$ is about $1 / 4$ to $1 / 2$, with $\Delta_{0}$ the CFL gap parameter for $M_{s}=0$. Here, $\Delta / \Delta_{0}$ for the CubeX crystal structure somewhat larger than that for the 2 Cube $45 \mathrm{z}$ structure and $\Delta / \Delta_{0}$ is a slowly increasing function of $M_{s}^{2} / \mu$, meaning a slowly decreasing function of density [20]. It is reasonable to estimate that $\Delta_{0}$ is between 10 and $100 \mathrm{MeV}$, but if $\Delta_{0}$ is in the upper half of this range then quark matter at accessible densities is likely in the CFL phase, rather than in the crystalline phase. So, we suggest that in interpreting (105) it is reasonable to estimate that

$$
5 \mathrm{MeV} \lesssim \Delta \lesssim 25 \mathrm{MeV},
$$

keeping in mind that a part of the uncertainty encompassed by this range comes from our lack of knowledge of $\Delta_{0}$ and a part comes from the $M_{s}^{2} / \mu$ dependence of $\Delta / \Delta_{0}$ described in Ref. [20]. The estimates (107) and (106) mean that our result (105) implies

$$
0.47 \mathrm{MeV} / \mathrm{fm}^{3}<\nu_{\mathrm{CQM}}<24 \mathrm{MeV} / \mathrm{fm}^{3} .
$$

We shall take this as an estimate of the magnitude of $\nu_{\mathrm{CQM}}$, although (105) is a better representation of our result for use in future work.

One qualitative way to appreciate how rigid the crystalline phases of quark matter are is to calculate the (anisotropic) velocities of long wavelength transverse and longitudinal phonons, as we have done for a few directions of propagation in the CubeX and 2 Cube 45 z crystal structures in Sections IV C and IV $\mathrm{D}$ respectively. We find that the transverse modes, whose restoring forces are governed by the shear moduli, propagate with velocities that are comparable to the velocity of longitudinal phonons.

To appreciate more quantitatively how rigid the crystalline phases of quark matter prove to be, we compare the shear modulus that we have calculated to that for the standard neutron star crust, which is a conventional crystal of positively charged ions immersed in a fluid of electrons (and, at sufficient depth, a fluid of neutrons). The shear modulus of this solid can be expressed as 62]

$$
\nu_{\mathrm{NM}}=c \frac{n_{i}(Z e)^{2}}{a},
$$

where $n_{i}$ is the number density of ions in the crust, $Z$ is the atomic number of the positively charged ions, $a=\left(3 /\left(4 \pi n_{i}\right)\right)^{1 / 3}$ is the average inter-ion spacing, $e^{2} \simeq 4 \pi / 137$ and $c \sim 0.1-0.2$ is a dimensionless constant. Because the crust is electrically neutral, the number density of ions is related to $n_{e}$, the electron number density, by $n_{i}=n_{e} / Z$. And, $n_{e}$ is given in terms of the mass and electric chemical potential $\mu_{e}$ of the electrons by

$$
n_{e}=\frac{\left(\mu_{e}^{2}-m_{e}^{2}\right)^{3 / 2}}{3 \pi^{2}},
$$

where $\mu_{e}$ is estimated to be in the range $20-80 \mathrm{MeV}$ and $Z \sim 40-50[62$. Using these estimates, we find

$$
0.092 \mathrm{keV} / \mathrm{fm}^{3}<\nu_{\mathrm{NM}}<23 \mathrm{keV} / \mathrm{fm}^{3} \text {. }
$$

Comparing to (108), we see that crystalline quark matter is more rigid than the conventional neutron star crust by at least a factor of 20 , and possibly by about three orders of magnitude.

We conclude that crystalline color superconducting quark matter is a very good solid indeed, which is remarkable since it is at the same time superfluid.

\section{B. Toward pulsar glitch phenomenology}

As discussed in the Introduction, the glitches that are observed to interrupt the gradual spin-down of spinning neutron stars are thought to arise from the sudden unpinning of an array of rotational vortices 
that had been pinned in place, at a fixed area density and hence a fixed angular momentum, while the other components of the star and in particular the observed surface had been gradually slowing down. When the stressed vortices unpin and separate, the superfluid component loses angular momentum while the surface spins up. Can these phenomena originate within crystalline color superconducting quark matter in the core of a neutron star? This phase of matter is a superfluid while at the same time having a rigid spatial modulation of its superfluid condensate, as we have seen. Understanding whether this makes it a plausible locus for the origin of pulsar glitches requires addressing three questions: Is crystalline color superconducting quark matter rigid enough? Do vortices in this phase of matter get pinned? And, how rapidly can angular momentum be transferred from a crystalline quark matter core that has just glitched to the outer crust whose surface is observed?

Our calculation constitutes an affirmative answer to the first question. We have shown that both the CubeX and 2Cube $45 z$ crystal structures have shear moduli with magnitude (105) which are 20 to 1000 times greater than those of the conventional neutron star crust within which glitches have long been assumed to originate.

Next, do vortices in fact get pinned? With what pinning force? This is a much harder question to address quantitatively because doing so requires going beyond the long wavelength phonon effective action. The question is what is the difference in the energy per unit length of a vortex centered on a nodal plane (or at the intersection of two nodal planes) of the condensate and one centered half way between neighboring nodal planes. Understanding this quantitatively requires constructing a vortex solution in the crystalline background, which is a challenging task. In the conventional neutron star crust, a vortex in a neutron superfluid is pinned on "impurities" embedded in the superfluid, namely the lattice of positively charged nuclei. In rotating crystalline color superconducting quark matter, the vortices are deformations of the phase and magnitude of the same condensate whose underlying magnitude modulation is the origin of the pinning. Unlike in the case of the shear modulus, which describes the response to a stress on length scales long compared to those characteristic of the crystal itself, the deformations introduced by a vortex will occur on length scales comparable to the lattice spacing of the un- derlying crystal. This means that constructing the vortices must be done self-consistently with analyzing the crystal structure itself — the pinning sites are in no sense extraneous impurities. We can provide a crude estimate of the pinning force, but we defer a quantitative response to this challenge to future work.

To estimate the pinning force, let us suppose (contrafactually) that the core radius of a vortex $\xi \sim 1 / \Delta$ is much smaller than the spacing between nodal planes of the crystalline condensate. If such a vortex is located where the underlying crystalline condensate is maximal, it will have to deform that condensate maximally since at the center of the vortex the condensate must vanish. Clearly, it will be energetically advantageous to locate the vortex at the intersection of nodal planes where the condensate already vanishes in the absence of a vortex. This argument translates into a pinning energy per unit length given at the level of dimensional analysis by

$$
\frac{E_{p}}{\ell}=f\left|\Omega_{\text {crystalline }}\right| \xi^{2}
$$

where $\left|\Omega_{\text {crystalline }}\right|$ is the condensation energy of the crystalline phase and where $f$ is some dimensionless factor. The corresponding pinning force per unit length is given by

$$
\frac{F_{p}}{\ell}=\frac{f\left|\Omega_{\text {crystalline }}\right| \xi^{2}}{b},
$$

where the length scale $b$ is half the spacing between neighboring nodal planes and hence one quarter of the lattice spacing. In both the CubeX and 2 Cube $45 z$ crystals, $b=\pi \sqrt{3} /(4 q)=1.13 / \delta \mu$. Recalling that $\delta \mu=M_{s}^{2} /(8 \mu)$, we can get a sense of the scale of $b$ by seeing that $b=18,12 \mathrm{fm}$ for $M_{\mathrm{c}}^{2} / \mu=100,150 \mathrm{MeV}$. Reading from plots in Ref. [20], we see that for $\Delta_{0}=25 \mathrm{MeV}$ this range of $M_{s}^{2} / \mu$ corresponds to a robust crystalline phase with $\left|\Omega_{\text {crystalline }}\right| \sim 2 \times 10^{5} \mathrm{MeV}^{4}$ and $\Delta \sim 5-10 \mathrm{MeV}$ if the crystal has the 2 Cube $45 \mathrm{z}$ structure or $\Delta \sim$ $10-15 \mathrm{MeV}$ if the crystal has the CubeX structure. We immediately see that $\xi=1 / \Delta$ and $b$ are comparable length scales, which makes this analysis unreliable at a quantitative level. One way of saying this is that the dimensionless factor $f$ must then be very much less than one, since the energy benefit by moving the vortex by a distance $b$ is of or$\operatorname{der}\left|\Omega_{\text {crystalline }}\right| \xi^{2}$ only if making this move shifts the core from a place where the condensate was maximal within the core area $\xi^{2}$ to a place where it is close 
to vanishing. A calculation of $f$ in the case where $\xi \sim b$ as is relevant in our context requires a quantitative analysis, but it is clear that the energy benefit of moving the vortex by a distance $b$ must then be
$\ll\left|\Omega_{\text {crystalline }}\right| \xi^{2}$. Putting the pieces together, we can write an estimate of the pinning force per unit length as

$$
\frac{F_{p}}{\ell}=0.7 \frac{\mathrm{MeV}}{(10 \mathrm{fm})^{2}}\left(\frac{f}{0.01}\right)\left(\frac{\left|\Omega_{\text {crystalline }}\right|}{2 \times 10^{5} \mathrm{MeV}^{4}}\right)\left(\frac{\xi}{20 \mathrm{fm}}\right)^{2}\left(\frac{15 \mathrm{fm}}{b}\right),
$$

where our choice of $f \sim 0.01$ as a fiducial value is a pure guess and the dependence of the other quantities in the estimate (114) on $\Delta_{0}$ and $M_{s}^{2} / \mu$ can be obtained from the results of Ref. [20], with the fiducial values we have used being reasonable for $\Delta_{0}=25 \mathrm{MeV}$ and $M_{s}^{2} / \mu=100-150 \mathrm{MeV}$.

We can compare our estimate (114) to the pinning force on neutron vortices in a conventional crust [63], in which neutron superfluid vortices are pinned on nuclei [45, 46, 47, 48, 49, 50, 51, 52, 53, 54, 55, 56, 57]. The pinning energy of a vortex per ion on which it is pinned is $E_{p}^{\mathrm{NM}} \sim 1-3 \mathrm{MeV}$ [46, 47, 48], the ions are spaced by a lattice spacing $b_{\mathrm{NM}} \sim 25-50 \mathrm{fm}[48]$, and the superfluid vortices have core radii $\xi_{\mathrm{NM}} \sim$ $4-20 \mathrm{fm}$ 48. Hence, the pinning force per unit length is [47, 48]

$$
\frac{E_{p}^{\mathrm{NM}}}{b_{\mathrm{NM}} \xi_{\mathrm{NM}}}=\frac{1-3 \mathrm{MeV}}{(25-50 \mathrm{fm})(4-20 \mathrm{fm})} .
$$

Although our estimate (114) is quite uncertain, given that we have not constructed the vortex solutions for rotating crystalline color superconducting quark matter, it seems reasonable to estimate that the pinning force per unit length on vortices within a putative crystalline quark matter neutron star core is comparable to that on neutron superfluid vortices within a conventional neutron star crust.

Recent calculations of the profile of vortices in BCS-paired superfluid gases of ultracold fermionic atoms 64] may make it easier to estimate the pinning force on vortices in the crystalline quark matter phase. In the cold atom context, it turns out that the radius of the vortex core is much smaller than the correlation length $\xi \sim 1 / \Delta$ which controls the long distance form of the vortex profile. Instead, the vortex core radius is $\sim 1 / k_{F}$, controlled by the Fermi momentum rather than by the correlation length. If this result were to be obtained in our context, it could mean replacing $\xi$ in (114) by $(1 / \mu) \sim 0.5 \mathrm{fm}$, reducing our fiducial estimate (114) by a factor of 1600. However, if the vortex cores do turn out to be as narrow as this then the assumption with which we began our estimate, namely that the core size is much less than the lattice spacing, becomes factual rather than contrafactual. This would mean that there is no longer any reason to expect the dimensionless factor $f$ to be much smaller than 1 , and would considerably reduce the uncertainty in the estimate. Replacing $f$ by 1 would increase the estimate (114) by a factor of 100, resulting in a pinning force which is only slightly smaller that on the superfluid neutron vortices within a conventional neutron star crust. We leave the determination of the profile of vortices in crystalline quark matter to future work, but it will clearly be very interesting to see whether they have narrow cores as in Ref. 64], and if so whether their pinning turns out to be controlled by their core radii or by the correlation length.

The third question which must be addressed is how, and how quickly, angular momentum can be transferred from a crystalline quark matter core to the outer crust. Some glitches are known to occur on timescales of minutes which means that if a glitch occurs within the core angular momentum must be transferred to the observed crust at least this fast. The core and crust are linked via being bathed in the same electron fluid and via magnetic fields. In the conventional glitch scenario, when the neutron superfluid in the crust suddenly slows down as its vortices come unpinned and the nonsuperfluid component of the crust, which includes the ions and the electrons, speeds up, the electron fluid couples the crust to the core well enough that the core also speeds up within seconds [49, 65]. We therefore expect that if a glitch occurs within a crystalline quark matter core, with this superfluid component slow- 
ing down, and if moving vortices can impart angular momentum to the electrons, then the electron fluid will ensure that the entire rest of the star including the outer crust speeds up. In the conventional scenario, the mechanism by which moving vortices exert a torque on the ions, and hence the electrons, in the crust has been described in Refs. [51, 52, 53]. In our case, we have not demonstrated how moving vortices in the crystalline phase can torque up the fluid of gapless charged quark quasiparticles, and hence the electrons.

So, tallying the status of the three questions that must be addressed: The first is settled, answered in the affirmative by our calculation of the shear modulus of the crystalline phase. The second remains to be addressed quantitatively but seems to be answered in the affirmative by our crude estimate of the pinning force on vortices in the crystalline phase. The third remains open, not yet addressed in a satisfactory fashion but nevertheless with no reason to doubt that its answer is also affirmative. Addressing the third question and addressing the second question quantitatively both require constructing the rotational vortex solutions for rotating crystalline quark matter. This is therefore the crucial remaining step in completing the connection between the microphysics of crystalline color superconducting quark matter and the phenomenology of pulsar glitches, and hence determining whether the characteristics of observed glitches rule out, or are consistent with, the presence of crystalline color superconducting quark matter within neutron stars.

It is also worth asking whether a "core-quake" scenario could be a viable model of glitches, qualitatively distinct from that based upon vortex pinning [40, 66, 67]. As a spinning neutron star slows down, it becomes less oblate. This will require macroscopic adjustments to the shape of a putative crystalline quark matter core. Given the enormous shear moduli of this rigid phase of matter, enormous amounts of elastic energy would be stored as the core is deformed and stressed, energy which would be released in core-quakes during which the crystalline core "breaks" and rearranges its structure so as to reduce its moment of inertia, consequently increasing its angular velocity. The original "crust-quake" model for pulsar glitches 68] failed because it failed to describe the magnitude and frequency of glitches in the Vela pulsar [48, 54, 55, 57]. Now that we know that crystalline quark matter has shear moduli which are 20 to 1000 times larger than those of the crust, core-quakes are worth re-investigating.

Finally, the advent of gravity wave detectors opens a new possibility for unique signatures of the presence of rigid matter within neutron stars, independent of transient phenomena like glitches [69]. LIGO has already set limits on the steady-state gravity wave emission from 78 nearby pulsars 70 , and these limits will become more stringent. Owen's work 69. shows that if an entire neutron star were solid, with a shear modulus as large as that we find for crystalline color superconducting quark matter, it could in principle support a quadrupole moment large enough such that the resulting gravity waves would already have been detected. This suggests that as the observational upper limits improve, the size of a putative rigid crystalline color superconducting quark matter core could be constrained. However, Owen's estimates for a star that is rigid in its entirety cannot be applied straightforwardly to the case where there is a rigid core surrounded by a fluid "mantle". Oblateness about the rotational $z$-axis is not enough to generate gravity waves; the quadrupole moment must be mis-aligned, such that the moment of inertia tensor satisfies $I_{x x} \neq I_{y y}$. It is hard to imagine how this could come about for a rigid quark matter core, whose shape will equilibrate to follow an equipotential surface via converting core material into mantle material or vice versa as needed at different locations along the core/mantle interface. Nevertheless, this line of enquiry warrants careful investigation.

\section{Acknowledgments}

We thank M. Alford, J. Bowers, E. Gubankova, B. Link, C. Manuel, B. Owen, D. Son and R. Xu for useful discussions. The work of MM has been supported by the "Bruno Rossi" fellowship program. The authors acknowledge the hospitality of the $\mathrm{Nu}$ clear Theory Group at LBNL. This research was supported in part by the Office of Nuclear Physics of the Office of Science of the U.S. Department of Energy under contract \#DE-AC02-05CH11231 and cooperative research agreement \#DF-FC02-94ER40818.

\section{APPENDIX A: PHONON MASS IS ZERO TO ALL ORDERS IN $\Delta$}

In this Appendix, we show explicitly that there cannot be any term in the phonon effective action 
at any order in $\Delta$ and at any order in $\mathbf{u}_{I}(x)$ which is nonzero if $\mathbf{u}_{I}(x)$ is constant over space and time, other than a trivial constant. Equivalently, we show that if we expand the Lagrangian density as a power series in $\mathbf{u}_{I}(x)$, every term has at least one derivative acting on each $\mathbf{u}_{I}(x)$, meaning that the mass of the phonons is zero. This is guaranteed by Goldstone's theorem, but the explicit demonstration of this "obvious" result is nontrivial and for this reason we give it here.

Before proceeding, we recall Eqs. (25) and (26), which imply that

$$
\Delta_{I}^{u}(x)=\Delta_{I} \sum_{\mathbf{q}_{I}^{a}} \exp \left(2 i \mathbf{q}_{I}^{a} \cdot\left(\mathbf{r}-\mathbf{u}_{I}(x)\right)\right.
$$

Since we want to prove the result to arbitrary powers in the $\mathbf{u}$ fields, we do not make an expansion in small $\mathbf{u}$. In terms of Feynman diagrams, this means that in evaluating the effective action in Eq. (43) we resum the vertices with increasing powers of $\mathbf{u}_{I}$. Therefore any vertex for $\Delta_{I}$ comes with a factor $\varepsilon_{I \alpha \beta} \varepsilon_{I i j} \exp \left(-2 i \mathbf{q}_{I}^{a} \cdot \mathbf{u}_{I}(x)\right)$ and a momentum insertion $2 \mathbf{q}_{I}^{a}$. On the other hand, the vertex for $\Delta_{I}^{*}$ comes with a vertex factor $\varepsilon_{I \alpha \beta} \varepsilon_{I i j} \exp \left(2 i \mathbf{q}_{I}^{a} \cdot \mathbf{u}_{I}(x)\right)$ and a momentum insertion $-2 \mathbf{q}_{I}^{a}$.

Integrating out the $\chi$ fields in Eq. (43) is equivalent to calculating all possible one-fermion-loop diagrams with arbitrarily many external phonon insertions. (Higher loop diagrams with internal phonon propagators are suppressed by powers of $\Lambda_{\mathrm{IR}} / \Delta$, with $\Lambda_{\mathrm{IR}}$ the typical energy of the phonon fields, and are therefore completely negligible.) A generic oneloop diagram will have $n_{I}$ vertices proportional to $\Delta_{I} \exp \left(-2 i \mathbf{q}_{I}^{a_{\kappa}} \cdot \mathbf{u}_{I}(x)\right)$ at which momenta $2 \mathbf{q}_{I}^{a_{\kappa}}$ are inserted into the loop, and $n_{I}$ vertices proportional to $\Delta_{I}^{*} \exp \left(2 i \mathbf{q}_{I}^{a_{\tau}} \cdot \mathbf{u}_{I}(x)\right)$ at which momenta $-2 \mathbf{q}_{I}^{a_{\tau}}$ are inserted into the loop. Here, $\kappa=1,2,3 \ldots n_{I}$ and $\tau=1,2,3 . . n_{I}$. The number of appearances of $\Delta_{I}$ has to be the same as that of $\Delta_{I}^{*}$ because the diagram can only depend on powers of $\left|\Delta_{I}\right|^{2}$. Different oneloop diagrams correspond to different choices of $n_{1}$, $n_{2}$ and $n_{3}$ and different choices of the $\mathbf{q}_{I}^{a_{\kappa}}$ 's and the $\mathbf{q}_{I}^{a_{\tau}}$ 's from among the sets $\left\{\mathbf{q}_{I}\right\}$. The color and flavor indices for the propagators in the diagram linking the vertices into a loop are chosen to be consistent with the color and flavor epsilon symbols associated to each vertex. The contribution from a generic oneloop diagram will be

$\mathcal{I} \propto\left|\Delta_{1}\right|^{2 n_{1}}\left|\Delta_{2}\right|^{2 n_{2}}\left|\Delta_{3}\right|^{2 n_{3}} \times \operatorname{Tr}\left[\frac{1}{i \not \partial+\mu_{j 1}} e^{2 \mathbf{q}_{1}^{a_{1}} \cdot\left(\mathbf{r}-\mathbf{u}_{1}(x)\right)} \frac{1}{i \not \partial-\mu_{j 2}} e^{-2 \mathbf{q}_{2}^{a_{1}} \cdot\left(\mathbf{r}-\mathbf{u}_{2}(x)\right)} \frac{1}{i \not \partial+\mu_{j 3}} e^{2 \mathbf{q}_{1}^{a_{2}} \cdot\left(\mathbf{r}-\mathbf{u}_{1}(x)\right)} \ldots\right]$.

We are interested in evaluating only the contribution from such a diagram in which no derivatives act on any $\mathbf{u}_{I}$ fields. This contribution is given by

$$
\begin{aligned}
\mathcal{I} \propto\left|\Delta_{1}\right|^{2 n_{1}}\left|\Delta_{2}\right|^{2 n_{2}}\left|\Delta_{3}\right|^{2 n_{3}} \times \operatorname{Tr} & \exp \left\{i \sum_{I} 2 \mathbf{u}_{I}(x) \cdot\left(\sum_{a=a_{1}}^{a_{n_{I}}} \mathbf{q}_{I}^{a}-\sum_{b=b_{1}}^{b_{n_{I}}} \mathbf{q}_{I}^{b}\right)\right\} \\
& \left.\times \frac{1}{i \not \partial+\mu_{j 1}} e^{2 \mathbf{q}_{1}^{a_{1}} \cdot \mathbf{r}} \frac{1}{i \not \partial-\mu_{j 2}} e^{-2 \mathbf{q}_{2}^{a_{1}} \cdot \mathbf{r}} \frac{1}{i \not \partial+\mu_{j 3}} e^{2 \mathbf{q}_{1}^{a_{2}} \cdot \mathbf{r}} \ldots\right] .
\end{aligned}
$$

Momentum conservation implies that the net momentum added to the loop is zero, i.e.

$$
\sum_{I}\left(\sum_{a=a_{1}}^{a_{n_{I}}} \mathbf{q}_{I}^{a}-\sum_{b=b_{1}}^{b_{n_{I}}} \mathbf{q}_{I}^{b}\right)=0
$$

(As we argued after Eq. (54), the momenta contributed by $\phi$ is much smaller than $\left|\mathbf{q}_{I}\right|$.)
We now recall from Section II that the magnitude of the $\mathbf{q}_{I}$ are different for different $I . \quad\left(\left|\mathbf{q}_{2}\right|=\eta \delta \mu_{2}\right.$ is close in value to $\left|\mathbf{q}_{3}\right|=\eta \delta \mu_{3}$, but they are not exactly equal because $\delta \mu_{2}$ and $\delta \mu_{3}$ differ by terms of order $M_{s}^{4} / \mu^{3}$.) This means that the momentum 
conservation condition (A4) can only be satisfied if

$$
\sum_{a=a_{1}}^{a_{n_{I}}} \mathbf{q}_{I}^{a}-\sum_{b=b_{1}}^{b_{n_{I}}} \mathbf{q}_{I}^{b}=0
$$

separately for each I. This implies that in Eq. (A3), the coefficients of each of the $\mathbf{u}_{I}$ cancel, and thus implies that Eq. (A3), the contribution of a generic one-loop diagram in which no derivatives act on any $\mathbf{u}_{I}$ 's, is independent of $\mathbf{u}_{I}$, making it a trivial con- stant in the phonon effective action. The phonons are therefore massless to all orders in $\Delta$ and $\mathbf{u}_{I}$.

As a special case, we can now demonstrate Eq. (51) explicitly. That is, we can show explicitly that $-\frac{1}{4 G} \int d^{4} x \operatorname{tr}_{C F}\left(\left(\Delta_{C F}^{u}\right)^{\dagger} \Delta_{C F}^{u}\right)$ is independent of $\mathbf{u}_{I}$ as must be the case since it includes no derivatives acting on $\mathbf{u}_{I}$, and so would constitute a mass term for the $\mathbf{u}_{I}$ if it were to depend on the $\mathbf{u}_{I}$. Indeed,

$$
\begin{aligned}
-\frac{1}{4 G} \int d^{4} x \operatorname{tr}_{C F}\left(\left(\Delta_{C F}^{u}\right)^{\dagger} \Delta_{C F}^{u}\right) & =-\frac{1}{G} \int d^{4} x \sum_{I}\left(\Delta_{I} \Delta_{I}^{*}\right) \sum_{\mathbf{q}_{I}^{a}} \sum_{\mathbf{q}_{I}^{b}} e^{2 \mathbf{q}_{I}^{a} \cdot\left(\mathbf{r}-\mathbf{u}_{I}(x)\right)} e^{-2 \mathbf{q}_{I}^{b} \cdot\left(\mathbf{r}-\mathbf{u}_{I}(x)\right)} \\
& =-(V T) \frac{1}{G} \sum_{I}\left(\Delta_{I} \Delta_{I}^{*}\right) P_{I},
\end{aligned}
$$

as given in (51).

\section{APPENDIX B: SINGLE PLANE WAVE}

In this Appendix, we investigate phonons in the presence of a condensate for which only one of the three $\Delta_{I}$ is nonzero, meaning that only quarks with two different colors and flavors form Cooper pairs. We take $\Delta_{3} \neq 0$ and $\Delta_{1}=\Delta_{2}=0$. This implies that there is pairing only between $u r$ and $d g$ quarks, and between $u g$ and $d r$ quarks, where $r, g$ and $b$ refer to the colors of the quarks. Furthermore, we assume that the set $\left\{\mathbf{q}_{3}\right\}$ contains only one vector, $\mathbf{q}$, so that $\Delta_{3}(x)$ varies in space as a single plane wave, $\Delta_{3}(\mathbf{r})=\Delta_{3} \exp (2 i \mathbf{q} \cdot \mathbf{r})$. In this simple case it is possible to derive the phonon effective action and the shear moduli without employing the GinzburgLandau expansion, working to order $\phi^{2}$.

The oscillation of the condensate is described by a single phonon field, $\mathbf{u}_{3}(x)$. In analogy with Eq. (26), we define

$$
\begin{aligned}
\Delta_{3}^{u}(x) & =\Delta_{3} e^{2 i \mathbf{q} \cdot\left(\mathbf{r}-\mathbf{u}_{3}(x)\right)} \\
& \approx \Delta_{3} e^{2 i \mathbf{q} \cdot \mathbf{r}}\left(1-i \phi(x)-\frac{1}{2} \phi(x)^{2}\right)
\end{aligned}
$$

where $\phi(x)=2 \mathbf{q} \cdot \mathbf{u}_{3}(x)$.

This condensate breaks translational symmetry in the $\hat{\mathbf{q}}$ direction, but leaves an $O(2)$ symmetry cor- responding to rotations about the $\hat{\mathbf{q}}$-axis unbroken. Taking $\hat{\mathbf{q}}$ along the $z$ axis, the potential energy $\mathcal{U}$ of Eqs. (82) and 84 must be symmetric under rotations about the $z$-axis, taking the form

$$
\begin{aligned}
\mathcal{U}= & \frac{\lambda^{z z z z}}{2}\left(s^{z z}\right)^{2} \\
& +\frac{\lambda^{x z x z}}{2}\left(\left(s^{x z}\right)^{2}+\left(s^{z x}\right)^{2}+\left(s^{y z}\right)^{2}+\left(s^{z y}\right)^{2}\right)
\end{aligned}
$$

where the strain tensors $s^{m u}$ are defined in (83). $\lambda^{z z z z}$ and $\lambda^{x z x z}=\lambda^{y z y z}$ are the two independent elastic moduli that we will now evaluate.

In writing the Lagrangian in terms of the NambuGorkov fields, we ignore the quarks that do not participate in pairing. We also note that the inverse propagator can be written as a block diagonal matrix made up of four blocks that correspond to the ur particles and $d g$ holes, $d g$ particles and ur holes, $u g$ particles and $d r$ holes and $d r$ particles and $u g$ holes. Since only quarks that belong to the same block interact, the inverse Nambu-Gorkov propagator can be written as

$$
S^{-1}=S_{u r-d g}^{-1} \oplus S_{d g-u r}^{-1} \oplus S_{u g-d r}^{-1} \oplus S_{d r-u g}^{-1},
$$

with 


$$
\begin{aligned}
& S_{u r-d g}^{-1}=\left(\begin{array}{cc}
i \not \partial+\mu_{u} & \left(C \gamma^{5}\right) \Delta_{3}^{u}(x) \\
-\left(C \gamma^{5}\right) \Delta_{3}^{u *}(x) & \left(i \not \partial-\mu_{d}\right)^{T}
\end{array}\right), S_{d g-u r}^{-1}=\left(\begin{array}{cc}
i \not \partial+\mu_{d} & \left(C \gamma^{5}\right) \Delta_{3}^{u}(x) \\
-\left(C \gamma^{5}\right) \Delta_{3}^{u *}(x) & \left(i \not \partial-\mu_{u}\right)^{T}
\end{array}\right), \\
& S_{u g-d r}^{-1}=\left(\begin{array}{cc}
i \not \partial+\mu_{u} & -\left(C \gamma^{5}\right) \Delta_{3}^{u}(x) \\
\left(C \gamma^{5}\right) \Delta_{3}^{u *}(x) & \left(i \not \partial-\mu_{d}\right)^{T}
\end{array}\right), S_{d r-u g}^{-1}=\left(\begin{array}{cc}
i \not \partial+\mu_{d} & -\left(C \gamma^{5}\right) \Delta_{3}^{u}(x) \\
\left(C \gamma^{5}\right) \Delta_{3}^{u *}(x) & \left(i \not \partial-\mu_{u}\right)^{T}
\end{array}\right) .
\end{aligned}
$$

The phonon effective action is obtained by integrating out the Nambu-Gorkov fields, yielding the result

$$
\begin{aligned}
i \mathcal{S}[\mathbf{u}]=\log (Z[\mathbf{u}])= & -i \frac{1}{G}(V T)\left|\Delta_{3}\right|^{2} \\
& +2 \operatorname{Tr}_{\mathrm{ng}} \log \left(S_{u r-d g}^{-1}\right)
\end{aligned}
$$

where we have used the property that the contribu- tions from the four blocks, $S_{u r-d g}^{-1}, S_{u g-d r}^{-1}, S_{d r-u g}^{-1}$ and $S_{d g-u r}^{-1}$, are equal. Since the trace of an operator does not change upon making a unitary transformation, corresponding to a change of basis, we can simplify $S^{-1}$ by choosing a basis which gets rid of the $\exp (2 i \mathbf{q} \cdot \mathbf{r})$ and the $C \gamma^{5}$ appearing with $\Delta_{3}$. In the new basis,

$$
\begin{aligned}
S_{u r-d g}^{-1}= & \left(\begin{array}{cc}
e^{-i \mathbf{q} \cdot \mathbf{r}} & 0 \\
0 & \left(C \gamma^{5}\right) e^{i \mathbf{q} \cdot \mathbf{r}}
\end{array}\right)\left(\begin{array}{cc}
i \not \partial+\mu_{u} & \left(C \gamma^{5}\right) \Delta_{3}^{u}(x) \\
-\left(C \gamma^{5}\right) \Delta_{3}^{u *}(x) & \left(i \not \partial-\mu_{d}\right)^{T}
\end{array}\right)\left(\begin{array}{cc}
e^{i \mathbf{q} \cdot \mathbf{r}} & 0 \\
0 & \left(-C \gamma^{5}\right) e^{-i \mathbf{q} \cdot \mathbf{r}}
\end{array}\right) \\
& =\left(\begin{array}{cc}
i \not \partial+\not \phi+\mu_{u} & \Delta_{3}\left(1-i \phi-\frac{1}{2} \phi^{2}\right) \\
\Delta_{3}^{*}\left(1+i \phi-\frac{1}{2} \phi^{2}\right) & i \not \partial-\not q-\mu_{d}
\end{array}\right) .
\end{aligned}
$$

In this simple case, the inverse propagator in the absence of the phonons can be inverted. (This is why we do not need to resort to the Ginzburg Landau approximation.) Hence we separate $S_{u r-d g}^{-1}$ into $S_{u r-d g}^{-1}=S_{\Delta}^{-1}+\Sigma$ with

$$
S_{\Delta}^{-1}=\left(\begin{array}{cc}
i \not \partial+\not q+\mu_{u} & \Delta_{3} \\
\Delta_{3}^{*} & \left(i \not \partial-\not 1-\mu_{d}\right)
\end{array}\right)
$$

and

$$
\Sigma=\left(\begin{array}{cc}
0 & \Delta_{3}\left(-i \phi-\frac{1}{2} \phi^{2}\right) \\
\Delta_{3}^{*}\left(+i \phi-\frac{1}{2} \phi^{2}\right) & 0
\end{array}\right)
$$

Since we have rotated out the phase $\exp (2 i \mathbf{q} \cdot \mathbf{r})$ from $\Delta_{3}$, the off-diagonal components of $S_{\Delta}^{-1}$ do not depend on position anymore. Upon inverting $S_{\Delta}^{-1}$, one gets the full propagator for the fermions in the absence of phonons, to all orders in $\Delta$. This propagator is diagonal in momentum space and therefore can be written in a simple way employing the weakcoupling approximation and the HDET formalism (implemented as in Eqs. (59) and (60) or more formally as in Ref. [60]), yielding

$$
S_{\Delta}(p)=\frac{1}{D_{\Delta}(p)}\left(\begin{array}{cc}
\left(\tilde{V} \cdot p-\hat{\mathbf{v}} \cdot \mathbf{q}-\delta \mu_{3}\right) & -\Delta_{3} \\
-\Delta_{3}^{*} & \left(V \cdot p-\hat{\mathbf{v}} \cdot \mathbf{q}-\delta \mu_{3}\right.
\end{array}\right)
$$

where

$$
\begin{aligned}
D_{\Delta}(p)= & \left(\tilde{V} \cdot p-\hat{\mathbf{v}} \cdot \mathbf{q}-\delta \mu_{3}\right)\left(V \cdot p-\hat{\mathbf{v}} \cdot \mathbf{q}-\delta \mu_{3}\right) \\
& -\left|\Delta_{3}\right|^{2} .
\end{aligned}
$$




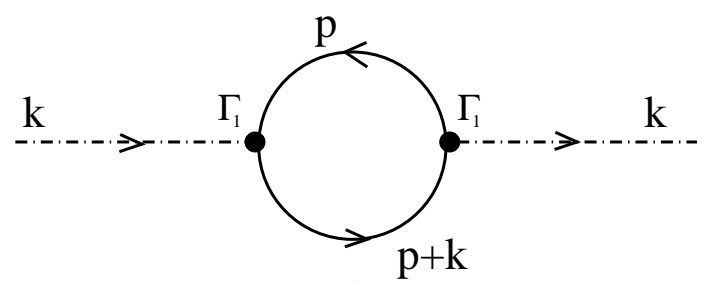

a)

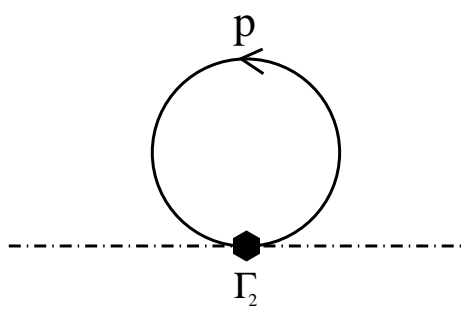

b)

FIG. 5: Diagrams contributing to the phonon self-energy. The dot-dashed lines correspond to the propagator of the phonon fields. Full lines correspond to the full propagator of quark quasi-particles. The phonon-quark-quark vertices $\Gamma_{1}$ and the phonon-phonon-quark-quark vertex $\Gamma_{2}$ are also shown. Diagram b) contributes only to the zero momentum polarization tensor. Diagram a) is nonvanishing for any value of the external momentum $k$ of the phonon.

We now consider the interaction with the phonon field. To second order in $\phi$, there are two interaction vertices, $i \phi(x) \Gamma_{1}$ and $-\frac{1}{2} \phi(x)^{2} \Gamma_{2}$, where

$$
\Gamma_{1}=\left(\begin{array}{cc}
0 & -\Delta_{3} \\
\Delta_{3}^{*} & 0
\end{array}\right) \text { and } \Gamma_{2}=\left(\begin{array}{cc}
0 & \Delta_{3} \\
\Delta_{3}^{*} & 0
\end{array}\right)
$$

The phonon effective action to order $\phi^{2}$ is given by the diagrams in Fig. 5 , namely

$$
\mathcal{S}^{\phi^{2}}=\operatorname{Tr}\left(S_{\Delta} \phi \Gamma_{1} S_{\Delta} \phi \Gamma_{1}\right)-\operatorname{Tr}\left(S_{\Delta} \phi^{2} \Gamma_{2}\right),
$$

where the trace is over Nambu-Gorkov indices and space-time. Evaluating the action along the same lines as in Section $\amalg$ IIE, we find

$$
\mathcal{S}^{\phi^{2}}=\frac{\mu^{2}}{\pi^{2}} \Delta_{3}^{2} \int \frac{d^{4} k}{(2 \pi)^{4}} \phi(k) \phi(-k) \int \frac{d p^{0}}{2 \pi i} \int_{-\Lambda}^{\Lambda} d s \int \frac{d \hat{\mathbf{v}}}{4 \pi} \frac{(V \cdot k)(\tilde{V} \cdot k)}{D_{\Delta}(p+k) D_{\Delta}(p)},
$$

where the $k$-independent second diagram in Fig. 5 has cancelled the $k$-independent contribution from the first diagram. We then integrate over $p_{0}$ and $\hat{\mathbf{v}}$ analytically and do the $s$-integral numerically. Upon returning to position space, the action takes the form (90) which, in this simple case, reduces to

$$
\mathcal{S}^{\phi^{2}}=\frac{1}{2} \int d^{4} x\left[\frac{\rho}{4 q^{2}}\left(\partial_{0} \phi(x)\right)^{2}-\frac{\kappa_{\perp}}{4 q^{2}}\left(\partial_{\perp} \phi(x)\right)^{2}-\frac{\kappa_{\|}}{4 q^{2}}\left(\partial_{\|} \phi(x)\right)^{2}\right],
$$

where $\partial_{\|} \equiv \hat{\mathbf{q}}(\hat{\mathbf{q}} \cdot \vec{\partial})$ and $\partial_{\perp} \equiv \vec{\partial}-\partial_{\|}$. We have written the factors of $4 q^{2}=4 \eta^{2} \delta \mu^{2}$ in the denominators in (B14) in order to facilitate comparison to (B2). The potential energy $\mathcal{U}$ in (B14) can easily be written in the form given in Eq. (B2) by substituting $\phi=2 \mathbf{q} \cdot \mathbf{u}_{3}$ and keeping only the term symmetric in $\partial_{i} \mathbf{u}_{j}$. Taking $\hat{\mathbf{q}}$ in the $z$ direction, one can easily see that $\lambda^{z z z z}=\lambda_{\|}$and $\lambda^{x z x z}=\lambda^{y z y z}=\lambda_{\perp}$.

The coefficients $\rho, \lambda_{\perp}$ and $\lambda_{\|}$are each proportional to $\mu^{2}$ and have nontrivial $\Delta_{3^{-}}$and $\delta \mu_{3^{-}}$ dependence. We have obtained them numerically for arbitrary values of $\Delta_{3}$, and by plotting them versus $\Delta_{3}^{2}$ at small $\Delta_{3}$ we have checked that $\rho$ and $\lambda_{\|}$are proportional to $\Delta_{3}^{2}$ and $\lambda_{\perp}$ is consistent with being proportional to $\Delta_{3}^{4}$. Within the accuracy of our numerical analysis, we find $\rho=\lambda_{\|}=\kappa$ to order $\Delta_{3}^{2}$, where $\kappa$ is given by (95). This is in agreement with what we obtain from the phonon effective action (93) evaluated to order $\Delta_{3}^{2}$ in the GinzburgLandau approximation, upon specializing (93) to 
the single plane wave case. It therefore provides a useful check on our calculations. Whether via the Ginzburg-Landau analysis of the main Sections of this paper or via the numerical all-order-in- $\Delta_{3}$ evaluation of this Appendix, we learn that $\lambda^{z z z z}=\kappa$ and $\lambda^{y z y z}=0$ to order $\Delta_{3}^{2}$, from which we conclude that the shear modulus of this single-plane-wave "crystal" is zero, to this order, and is presumably proportional to $\mu^{2} \Delta_{3}^{4} / \delta \mu^{2}$.
[1] J. C. Collins and M. J. Perry, Phys. Rev. Lett. 34, 1353 (1975)

[2] For reviews, see K. Rajagopal and F. Wilczek, arXiv:hep-ph/0011333, M. G. Alford, Ann. Rev. Nucl. Part. Sci. 51, 131 (2001) arXiv:hep-ph/0102047; G. Nardulli, Riv. Nuovo Cim. 25N3, 1 (2002) arXiv:hep-ph/0202037; S. Reddy, Acta Phys. Polon. B 33, 4101 (2002) arXiv:nucl-th/0211045; T. Schäfer, arXiv:hep-ph/0304281; D. H. Rischke, Prog. Part. Nucl. Phys. 52, 197 (2004) arXiv:nucl-th/0305030; M. Alford, Prog. Theor. Phys. Suppl. 153, 1 (2004) arXiv:nucl-th/0312007; M. Buballa, Phys. Rept. 407, 205 (2005) arXiv:hep-ph/0402234; H. c. Ren, arXiv:hep-ph/0404074; I. Shovkovy, arXiv:nucl-th/0410091 T. Schäfer, arXiv:hep-ph/0509068; M. Alford and K. Rajagopal, arXiv:hep-ph/0606157.

[3] J. Bardeen, L.N. Cooper, and J.R. Schrieffer, Phys. Rev. 108, 1175 (1957).

[4] M. G. Alford, K. Rajagopal and F. Wilczek, Nucl. Phys. B 537, 443 (1999) arXiv:hep-ph/9804403.

[5] M. Alford and K. Rajagopal, JHEP 0206, 031 (2002) arXiv:hep-ph/0204001.

[6] M. Alford, C. Kouvaris and K. Rajagopal, Phys. Rev. Lett. 92, 222001 (2004) arXiv:hep-ph/0311286.

[7] M. Alford, C. Kouvaris and K. Rajagopal, Phys. Rev. D 71, 054009 (2005) arXiv:hep-ph/0406137.

[8] M. Alford, C. Kouvaris and K. Rajagopal, arXiv:hep-ph/0407257.

[9] S. B. Rüster, I. A. Shovkovy and D. H. Rischke, Nucl. Phys. A 743, 127 (2004) arXiv:hep-ph/0405170.

[10] K. Fukushima, C. Kouvaris and K. Rajagopal, Phys. Rev. D 71, 034002 (2005) arXiv:hep-ph/0408322.

[11] M. Alford, P. Jotwani, C. Kouvaris, J. Kundu and K. Rajagopal, Phys. Rev. D 71, 114011 (2005) arXiv:astro-ph/0411560.

[12] H. Abuki, M. Kitazawa and T. Kunihiro, Phys. Lett. B 615, 102 (2005) arXiv:hep-ph/0412382.

[13] S. B. Rüster, V. Werth, M. Buballa, I. A. Shovkovy and D. H. Rischke, Phys. Rev. D 72, 034004 (2005) arXiv:hep-ph/0503184.

[14] I. Shovkovy and M. Huang, Phys. Lett. B 564, 205 (2003) arXiv:hep-ph/0302142; M. Huang and I. Shovkovy, Nucl. Phys. A 729, 835 (2003)
arXiv:hep-ph/0307273.

[15] E. Gubankova, W. V. Liu and F. Wilczek, $\begin{array}{lllll}\text { Phys. Rev. Lett. 91, } 032001 \quad \text { (2003) } & \end{array}$ arXiv:hep-ph/0304016.

[16] M. Huang and I. A. Shovkovy, Phys. Rev. D 70, 051501 (2004) arXiv:hep-ph/0407049; M. Huang and I. A. Shovkovy, Phys. Rev. D 70, 094030 (2004) arXiv:hep-ph/0408268; I. Giannakis and H. C. Ren, Phys. Lett. B 611, 137 (2005) arXiv:hep-ph/0412015; M. Alford and Q. h. Wang, J. Phys. G 31, 719 (2005) arXiv:hep-ph/0501078; M. Huang, Phys. Rev. D 73, 045007 (2006) arXiv:hep-ph/0504235.

[17] R. Casalbuoni, R. Gatto, M. Mannarelli, G. Nardulli and M. Ruggieri, Phys. Lett. B 605, 362 (2005) [Erratum-ibid. B 615, 297 (2005)] arXiv:hep-ph/0410401; K. Fukushima, Phys. Rev. D 72, 074002 (2005) arXiv:hep-ph/0506080].

[18] R. Casalbuoni, R. Gatto, N. Ippolito, G. Nardulli and M. Ruggieri, Phys. Lett. B 627, 89 (2005) arXiv:hep-ph/0507247.

[19] M. Mannarelli, K. Rajagopal and R. Sharma, Phys. Rev. D 73, 114012 (2006) arXiv:hep-ph/0603076.

[20] K. Rajagopal and R. Sharma, Phys. Rev. D 74, 094019 (2006) arXiv:hep-ph/0605316; K. Rajagopal and R. Sharma, J. Phys. G 32, S483 (2006) arXiv:hep-ph/0606066.

[21] A. Kryjevski, arXiv:hep-ph/0508180 T. Schafer, Phys. Rev. Lett. 96, $012305 \quad$ (2006) arXiv:hep-ph/0508190.

[22] A. Gerhold and T. Schafer, Phys. Rev. D 73, 125022 (2006) arXiv:hep-ph/0603257; A. Gerhold, T. Schaefer and A. Kryjevski, arXiv:hep-ph/0612181.

[23] E. V. Gorbar, M. Hashimoto and V. A. Miransky, Phys. Lett. B 632, 305 (2006) arXiv:hep-ph/0507303; E. V. Gorbar, M. Hashimoto, V. A. Miransky and I. A. Shovkovy, Phys. Rev. D 73, 111502 (2006) arXiv:hep-ph/0602251; E. V. Gorbar, M. Hashimoto and V. A. Miransky, arXiv:hep-ph/0701211

[24] M. Iwasaki and T. Iwado, Phys. Lett. B 350, 163 (1995). T. Schäfer, Phys. Rev. D 62, 094007 (2000) arXiv:hep-ph/0006034; M. Buballa, J. Hosek and M. Oertel, Phys. Rev. Lett. 90, 182002 (2003) arXiv:hep-ph/0204275; A. Schmitt, 
Q. Wang and D. H. Rischke, Phys. Rev. D 66, 114010 (2002) arXiv:nucl-th/0209050; M. G. Alford, J. A. Bowers, J. M. Cheyne and G. A. Cowan, Phys. Rev. D 67, 054018 (2003) arXiv:hep-ph/0210106; A. Schmitt, Phys. Rev. D 71, 054016 (2005) arXiv:nucl-th/0412033]; M. G. Alford and G. A. Cowan, J. Phys. G 32, 511 (2006) arXiv:hep-ph/0512104.

[25] K. Rajagopal and A. Schmitt, Phys. Rev. D 73, 045003 (2006) arXiv:hep-ph/0512043.

[26] M. G. Alford, K. Rajagopal and F. Wilczek, Phys. Lett. B 422, 247 (1998) arXiv:hep-ph/9711395.

[27] R. Rapp, T. Schafer, E. V. Shuryak and M. Velkovsky, Phys. Rev. Lett. 81, 53 (1998) arXiv:hep-ph/9711396.

[28] A. I. Larkin and Yu. N. Ovchinnikov, Zh. Eksp. Teor. Fiz. 47, 1136 (1964)[Sov. Phys. JETP 20, 762 (1965)]; P. Fulde and R. A. Ferrell, Phys. Rev. 135, A550 (1964); S. Takada and T. Izuyama, Prog. Theor. Phys. 41, 635 (1969).

[29] M. G. Alford, J. A. Bowers and K. Rajagopal, Phys. Rev. D 63, 074016 (2001) arXiv:hep-ph/0008208.

[30] J. A. Bowers and K. Rajagopal, Phys. Rev. D 66, 065002 (2002) arXiv:hep-ph/0204079.

[31] R. Casalbuoni, R. Gatto, M. Mannarelli and G. Nardulli, Phys. Lett. B 511, 218 (2001) arXiv:hep-ph/0101326; R. Casalbuoni, R. Gatto, M. Mannarelli and G. Nardulli, Phys. Rev. D 66, 014006 (2002) arXiv:hep-ph/0201059; R. Casalbuoni, E. Fabiano, R. Gatto, M. Mannarelli and G. Nardulli, Phys. Rev. D 66, 094006 (2002) arXiv:hep-ph/0208121; R. Casalbuoni, R. Gatto, M. Mannarelli, G. Nardulli, M. Ruggieri and S. Stramaglia, Phys. Lett. B 575, 181 (2003) arXiv:hep-ph/0307335; R. Casalbuoni, M. Ciminale, M. Mannarelli, G. Nardulli, M. Ruggieri and R. Gatto, Phys. Rev. D 70, 054004 (2004) arXiv:hep-ph/0404090.

[32] J. A. Bowers, J. Kundu, K. Rajagopal and E. Shuster, Phys. Rev. D 64, 014024 (2001) arXiv:hep-ph/0101067.

[33] A. K. Leibovich, K. Rajagopal and E. Shuster, Phys. Rev. D 64, 094005 (2001) arXiv:hep-ph/0104073.

[34] J. Kundu and K. Rajagopal, Phys. Rev. D 65, 094022 (2002) arXiv:hep-ph/0112206.

[35] M. Ciminale, G. Nardulli, M. Ruggieri and R. Gatto, Phys. Lett. B 636, 317 (2006) arXiv:hep-ph/0602180; K. Fukushima, Phys. Rev. D 73, 094016 (2006) arXiv:hep-ph/0603216.

[36] N. D. Ippolito, G. Nardulli and M. Ruggieri, arXiv:hep-ph/0701113.

[37] D. T. Son, Phys. Rev. Lett. 94, 175301 (2005) arXiv:cond-mat/0501658.

[38] A. F. Andreev and I. M. Lifshitz, Zh. Eksp. Teor. Fiz. 56, 2057 (1969) [Sov. Phys. JETP 29, 1107 (1969)]; G. V. Chester, Phys. Rev. A2, 256 (1970);
A. J. Leggett, Phys. Rev. Lett. 25, 1543 (1970).

[39] E. Kim and M. H. W. Chan, Nature 427, 225 (2004); E. Kim and M. H. W. Chan, Science 305, 1941 (2004).

[40] R. X. Xu, Astrophys. J. 596, L59 (2003) arXiv:astro-ph/0302165; A. Z. Zhou, R. X. Xu, X. J. Wu, N. Wang and X. Y. Hong, Astropart. Phys. 22, 73 (2004) arXiv:astro-ph/0404554; R. X. Xu, D. J. Tao and Y. Yang, Mon. Not. Roy. Astron. Soc. Lett. 373, L85 (2006) arXiv:astro-ph/0607106.

[41] R. Anglani, G. Nardulli, M. Ruggieri and M. Mannarelli, Phys. Rev. D 74, 074005 (2006) arXiv:hep-ph/0607341.

[42] N. Iwamoto, Phys. Rev. Lett. 44, 1637 (1980).

[43] For a recent analysis with references, see D. Page, J. M. Lattimer, M. Prakash and A. W. Steiner, Astrophys. J. Suppl. 155, 623 (2004) arXiv:astro-ph/0403657.

[44] Unlikely, but not resolved definitively. The calculation of Ref. [41] is a first step in the sense that it treats a very simple "crystal" structure in which the $\langle u d\rangle$ and $\langle u s\rangle$ condensates are each single plane waves. The neutrino emissivity of the CubeX and 2 Cube $45 z$ crystal structures remain to be calculated, meaning that it remains to be determined by how much the phase space for direct URCA reactions like $u+e \rightarrow d+\nu$ is reduced in these crystalline phases with pairing with many different wave vectors.

[45] P. W. Anderson and N. Itoh, Nature 256, 25 (1975).

[46] M. A. Alpar, Astrophys. J. 213, 527 (1977); M. A. Alpar, P. W. Anderson, D. Pines, and J. Shaham, Astrophys. J. 249, L29 (1981); P. W. Anderson, M. A. Alpar, D. Pines and J. Shaham, Phil. Mag. A, 45, 227 (1982).

[47] M. A. Alpar, P. W. Anderson, D. Pines, and J. Shaham, Astrophys. J. 276, 325 (1984).

[48] M.A. Alpar, P.W. Anderson, D. Pines and J. Shaham, Astrophys. J. 278, 791 (1984).

[49] M. A. Alpar, S. A. Langer and J. A. Sauls, Astrophys. J. 282, 533 (1988); M. A. Alpar and J. A. Sauls, Astrophys. J. 327, 723.

[50] M. A. Alpar, R. Nandkumar and D. Pines, Astrophys. J. 288, 191 (1985). M. A. Alpar, R. Nandkumar and D. Pines, Astrophys. J. 311, 197 (1986).

[51] R. I. Epstein and G. Baym, Astrophys. J. 387, 276 (1992).

[52] B. Link, R. I. Epstein and G. Baym, Astrophys. J. 403, 285 (1993).

[53] P. B. Jones, Mon. Not. R. Astron. Soc. 263, 619 (1993); P. B. Jones, Phys. Rev. Lett. 79, 792 (1997).

[54] M. A. Alpar, H. F. Chau, K. S. Cheng and D. Pines, Astrophys. J. 409, 345 (1993); M. A. Alpar, H. F. Chau, K. S. Cheng and D. Pines, Astrophys. J. 427, L29 (1994). 
[55] M. A. Alpar and A. Baykal, Mon. Not. R. Astron. Soc., 269, 849 (1994).

[56] M. A. Alpar, H. F. Chau, K. S. Cheng and D. Pines, Astrophys. J. 459, 706 (1996).

[57] For reviews see D. Pines and M. A. Alpar, Nature 316, 27 (1985); D. Pines in Neutron Stars: Theory and Observation, J. Ventura and D. Pines, eds., 57 (Kluwer, 1991); M. A. Alpar, in The Lives of Neutron Stars, M. A. Alpar et al., eds., 185 (Kluwer, 1995).

[58] A. Gerhold and A. Rebhan, Phys. Rev. D 68, 011502 (2003) arXiv:hep-ph/0305108; A. Kryjevski, Phys. Rev. D 68, 074008 (2003) arXiv:hep-ph/0305173; D. D. Dietrich and D. H. Rischke, Prog. Part. Nucl. Phys. 53, 305 (2004) arXiv:nucl-th/0312044; A. Gerhold, Phys. Rev. D 71, 014039 (2005) arXiv:hep-ph/0411086].

[59] R. Casalbuoni, M. Ciminale, R. Gatto, G. Nardulli and M. Ruggieri, Phys. Lett. B 642, 350 (2006) arXiv:hep-ph/0606242.

[60] G. Nardulli, in Ref. [2].
[61] L. D. Landau and E. M. Lifschitz, Theory of Elasticity, 3rd edition, Oxford, Pergamon (1981).

[62] T. Strohmayer, H. M. van Horn, S. Ogata, H. Iyetomi and S. Ichimaru, Astrophys. J. 375, 679 (1991).

[63] J. W. Negele and D. Vautherin, Nucl. Phys. A 207, 298 (1973).

[64] R. Sensarma, M. Randeria and T.-L. Ho, Phys. Rev. Lett. 96, 090403 (2006) arXiv:cond-mat/0510761.

[65] I. Easson, Astrophys. J. 228, 257 (1979).

[66] D. Pines, J. Shaham and M. Ruderman, Nature 237, 83 (1972); G. Baym, D. Q. Lamb and F. K. Lamb, Astrophys. J. 208, 829 (1976).

[67] B. Link, private communication.

[68] M. Ruderman, Nature 223, 597 (1969); G. Baym, and D. Pines, Ann. Phys. 66, 816 (1971).

[69] B. J. Owen, Phys. Rev. Lett. 95, 211101 (2005) arXiv:astro-ph/0503399.

[70] B. Abbott et al. [LIGO Scientific Collaboration], arXiv:gr-qc/0702039 